\title{
The Photoevaporative Wind from the Disk of TW Hya
}

\author{
I. Pascucci ${ }^{1}$ \\ Lunar and Planetary Laboratory, The University of Arizona, Tucson, AZ 85721, USA \\ pascucci@lpl.arizona.edu
}

M. Sterzik

European Southern Observatory, Casilla 19001, Santiago 19, Chile

R. D. Alexander

Department of Physics \& Astronomy, University of Leicester, University Road, Leicester, LE1 7RH, UK

S. H. P. Alencar

Departamento de Fisica - ICEx UFMG, MG, Brazil

U. Gorti ${ }^{2}$

SETI Institute, 189 Bernardo Ave., Mountain View, CA 94043, USA

D. Hollenbach

SETI Institute, 189 Bernardo Ave., Mountain View, CA 94043, USA

\begin{abstract}
J. Owen
Institute of Astronomy, Madingley Road, Cambridge CB3 0HA

B. Ercolano

University Observatory Munich, D-81679, Munich, Germany
\end{abstract}

\footnotetext{
${ }^{1}$ Department of Physics and Astronomy, Johns Hopkins University, Baltimore, MD 21218

${ }^{2}$ NASA Ames Research Center, Moffett Field, CA 94035, USA
} 


\begin{abstract}
S. Edwards
Astronomy Department, Smith College, Northampton, MA 01063 USA
\end{abstract}

Received __; accepted _

*Based on observations made with VISIR on the UT3/Melipal ESO Telescope at Paranal under programme ID 084.C-0088(A) 


\begin{abstract}
Photoevaporation driven by the central star is expected to be a ubiquitous and important mechanism to disperse the circumstellar dust and gas from which planets form. Here, we present a detailed study of the circumstellar disk surrounding the nearby star TW Hya and provide observational constraints to its photoevaporative wind. Our new high-resolution $(R \sim 30,000)$ mid-infrared spectroscopy in the [Ne II] $12.81 \mu \mathrm{m}$ line confirms that this gas diagnostic traces the unbound wind component within $10 \mathrm{AU}$ from the star. From the blueshift and asymmetry in the line profile, we estimate that most $(>80 \%)$ of the $[\mathrm{Ne}$ II] emission arises from disk radii where the midplane is optically thick to the redshifted outflowing gas, meaning beyond the 1 or $4 \mathrm{AU}$ dust rim inferred from other observations. We re-analyze high-resolution $(R \sim 48,000)$ archival optical spectra searching for additional transitions that may trace the photoevaporative flow. Unlike the [Ne II] line, optical forbidden lines from OI, SII, and MgI are centered at the stellar velocity and have symmetric profiles. The only way these lines could trace the photoevaporative flow is if they arise from a disk region physically distinct from that traced by the [Ne II] line, specifically from within the optically thin dust gap. However, the small $(\sim 10 \mathrm{~km} / \mathrm{s})$ FWHM of these lines suggest that most of the emitting gas traced at optical wavelengths is bound to the system rather than unbound. We discuss the implications of our results for a planet-induced versus a photoevaporation-induced gap.
\end{abstract}

Subject headings: accretion, accretion disks - infrared: stars - planetary systems: protoplanetary disks - stars: individual (TW Hya) 


\section{Introduction}

One of the yet unsolved questions in planet formation is how young stars lose their disks. Planet formation certainly contributes to the clearing of primordial disk material. Its first steps, the coagulation of sub-micron sized grains and their subsequent settling to the disk midplane, have been identified toward many protoplanetary disks (e.g., Natta et al. 2007 for a review). However, planet formation is likely not the major disk dispersal mechanism, as evinced by the small total mass in the planets (both in our solar system and in other planetary systems) when compared to the mass of protoplanetary disks (e.g., Hayashi et al. 1985). Furthermore, a disk dispersal mechanism based only on planet formation would require shorter dispersal timescales in high metallicity environments (Ercolano \& Clarke 2010), which is not supported by recent observations of disk fractions in low metallicity clusters of the extreme outer Galaxy (Yasui et al. 2009).

Current models of protoplanetary disk evolution suggest that viscous evolution (accretion of gas onto the central star) and photoevaporation driven by the central star (heating of disk gas to thermal escape velocities) are the main disk dispersal mechanisms (e.g., Hollenbach et al. 2000; Gorti et al. 2009; Owen et al. 2010). Indeed, there is abundant observational evidence that young stars are accreting disk gas: from the optical and ultraviolet excess continuum emission arising from the accretion shock (e.g., Calvet et al. 2000) to the broad permitted emission lines from infalling gas (e.g., Muzerolle et al. 1998). Measurements of mass accretion rates using these diagnostics are now available for hundreds of stars in nearby star-forming regions and qualitatively follow the time evolution predicted by these models (Hartmann et al. 1998; Sicilia-Aguilar et al. 2010). However, at least two other observables are not reproduced by models of viscously evolving disks. First, the disk dispersal timescale, which is measured to be of just a few Myr, is predicted to be too long using the viscosity inferred from mass accretion rate measurements. Both optically thick 
dust disks as well as gaseous disks should be numerous and detectable around stars that are 10 Myr-old, which contrasts with observations (e.g., Pascucci \& Tachibana 2010). Second, the transition from disk-bearing to disk-less stars is observed to be quick (just $\sim 10^{5}$ years), too quick to be explained by viscous evolution alone (see e.g., Armitage 2010 for a review).

These observables led theorists to propose photoevaporation driven by the central star as the next ubiquitous and most relevant mechanism to disperse protoplanetary disks (e.g., Hollenbach et al. 2000; Dullemond et al. 2007). In the first model combining the effect of accretion and photoevaporation (Clarke et al. 2001), extreme UV photons (EUV, $13.6 \mathrm{eV}<h \nu \leq 100 \mathrm{eV}$ ) from the central star heat and ionize the disk surface. Beyond the radius at which the sound speed of the gas equals the local Keplerian orbital speed (gravitational radius, $r_{\mathrm{g}}$ ), the hot gas becomes unbound and a photoevaporative wind is established. When the disk accretion rate through $r_{\mathrm{g}}$ drops below the wind loss rate, photoevaporation limits the supply of gas to the inner disk, which drains onto the star on the local viscous timescale - of order $10^{5}$ years. Many theoretical developments have been made since this first model. First, Liffman (2003), Font et al. (2004), and Adams et al. (2004) have included the effects of angular momentum support and showed that photoevaporation occurs within $r_{\mathrm{g}}$ and happens mostly outside $\sim 0.15 r_{\mathrm{g}}$ (hereafter critical radius). Second, Alexander et al. $(2006 \mathrm{a}, \mathrm{b})$ showed that the direct EUV irradiation of the disk rim can drastically reduce the disk lifetime once the inner disk is cleared out. Third, Ercolano et al. (2008), and Gorti \& Hollenbach (2009) have recognized that stellar Xrays and FUV photons can launch winds from denser disk regions and thus dramatically increase the wind mass-loss rate produced by EUV photons alone.

Recently, several papers have discussed whether other disk properties, beyond disk lifetimes, are consistent with photoevaporation models. There is yet no consensus: Kim et al. (2009) include photoevaporation as a possible explanation for some of the 
transition disks in their sample; Currie et al. (2009) argue that the large number of evolved dust disks at 5 Myr is inconsistent with photoevaporation; Cieza et al. (2008) find support for photoevaporation based on the lack of optically thin inner disks with detectable dust outer disks. All these studies and similar ones relied on comparing model predictions with the properties of dust disks. A more direct way to test photoevaporation is to observe the gas disk component, especially that component that is not gravitationally bound and is part of the photoevaporating flow.

Following this approach, Pascucci \& Sterzik (2009) have shown that the [Ne II] line at $12.81 \mu \mathrm{m}$ is a good diagnostic for the photoevaporative wind in some systems that have transition-like spectral energy distributions (SEDs). Such systems display small near-infrared excess emission but large mid- to far-infrared emission and are thus thought to be in the process of clearing out their primordial disk material. The most convincing case for photoevaporation is that for the almost face-on transition disk around the 10 Myr-old star TW Hya. The measured blueshift of $\sim 6 \mathrm{~km} / \mathrm{s}$ demonstrates that there is unbound gas leaving the disk and moving toward the observer (Pascucci \& Sterzik 2009). If the wind is fully ionized, the measured [Ne II] flux translates into a mass-loss rate of $\sim 10^{-10} \mathrm{M}_{\odot} / \mathrm{yr}$. However, if the wind is mostly neutral, as predicted by models of Xray photoevaporation 1 , then the $[\mathrm{Ne} \mathrm{II}]$ emission is a trace component of the wind and the observed line strength and profile imply a much larger wind rate up to $\sim 10^{-8} \mathrm{M}_{\odot} / \mathrm{yr}$ (Ercolano \& Owen 2010). We also note that this relatively old star is still accreting disk gas at a rate that is about an oder of magnitude lower than that of classical T Tauri stars $\left(\sim 10^{-9} \mathrm{M}_{\odot} / \mathrm{yr}\right.$, Muzerolle et al. 2000 and Alencar \& Batalha 2002).

\footnotetext{
${ }^{1}$ Gorti \& Hollenbach (2009) point out that Xrays alone cannot drive a strong wind but it is rather the combination of FUV photons (which dissociate the molecules and thereby reduce the ability of the disk gas to cool) and Xrays (which heat the gas) that drive the wind
} 
Being at only 51 pc (Mamajek 2005) and clearly presenting evidence for photoevaporation, TW Hya is the ideal target to provide additional observational constraints to models of disk photoevaporation and to help determine wind mass-loss rates. In this paper we present new observations of the [Ne II] $12.81 \mu \mathrm{m}$ line toward TW Hya and employ the spectroastrometry technique to estimate the radii traced by the ionized wind component. We also analyze archival optical spectra to search for the neutral disk wind component predicted by Xray-driven photoevaporation models. We discuss the implications of our results on the gas distribution in the inner disk of TW Hya and speculate on how that structure might link to planet formation and photoevaporation.

\section{Photoevaporative disk wind models}

Before presenting our observations and analyzing the infrared and optical spectra, we wish to briefly summarize the main features of photoevaporative disk wind models and point out their major differences. Our goal is to clarify what observables can be used to characterize the structure and extension of the wind based on model predictions.

There are basically three main sources of ionization and heating of the disk surface: Xrays $(0.1 \mathrm{keV}<h \nu<10 \mathrm{keV})$, EUV $(13.6 \mathrm{eV}<h \nu<0.1 \mathrm{keV})$, and FUV $(6 \mathrm{eV}<h \nu<$ $13.6 \mathrm{eV}$ ) photons. The first and simplest models assumed that photoevaporation is driven solely by stellar EUV photons. These photons ionize the hydrogen in the very upper layers of the disk and heat it to a temperature of $\sim 10^{4} \mathrm{~K}$, independent of radius, thus creating a kind of coronal H II region (Hollenbach et al. 1994, Alexander et al. 2006a,b). This fully ionized layer will produce forbidden lines at infrared and optical wavelengths whose relative intensities depend on the intensity and the spectral energy distribution of the EUV field impinging on the disk (Hollenbach \& Gorti 2009), which is not constrained observationally. Assuming plausible stellar ionizing fluxes of $\sim 10^{41}$ photons/s and a solar abundance of the 
elements, EUV-only models predict a relatively strong [Ne II] emission line at $12.81 \mu \mathrm{m}$ (Alexander 2008, Hollenbach \& Gorti 2009) and similarly strong [S II] lines at 6731 and $6716 \AA$ and [N II] lines at $6583 \AA$ (Font et al. 2004). Because the fraction of neutral gas is very low in the EUV layer, forbidden lines from atomic species, such as the [O I] line at $6300 \AA$, are expected to be weak (Font et al. 2004, Hollenbach \& Gorti 2009). The most recent developments of the EUV-only models include a detailed treatment of the flow structure and predict line profiles from the wind in addition to line intensities (Alexander 2008). As we will show in Sect. 4.4.1, these simple models can also produce channel maps at specific emission lines that can be used to constrain the spatial distribution of the wind.

More comprehensive models, such as those developed by Ercolano et al. and Gorti \& Hollenbach, include other sources of disk heating and ionization in addition to stellar EUV photons. Specifically, the most recent development of the Ercolano et al. models includes Xray and EUV heating and ionization of the atomic disk gas and a 2D hydrodynamic calculation wich allows predicting line intensities and profiles from a variety of forbidden and semi-permitted transitions in the wind (Ercolano \& Owen 2010). Hereafter, we will call these models X-EUV models. Gorti \& Hollenbach (2009) include all three main heating and ionization sources and a detailed treatment of molecular cooling in their thermal balance calculations. For this more complex model, which we will call XE-FUV model, hydrodynamical calculations have not been perfomed yet hence we will limit the comparison between model and observations to line intensities. The X-EUV and XE-FUV models further differ in their assumptions of the stellar Xray and EUV spectra and therefore also in the predicted mass-flow rates. Regardless of these differences, the inclusion of Xray and FUV heating results in a predominantly denser, but cooler (hence mainly neutral), photoevaporative wind compared to the EUV-only models and the mass loss rates are higher by one (XE-FUV) or two (X-EUV) orders of magnitude. Depending on the star/disk parameters, the $[\mathrm{OI}]$ emission line at $6300 \AA$, whose low-velocity component is always 
detected toward accreting T Tauri stars (Hartigan et al. 1995), may trace the neutral component of the disk wind (Ercolano \& Owen 2010). Other forbidden lines that are expected to be strong in the Xray ionized wind are the [Ne II] line at $12.81 \mu \mathrm{m}$ (Hollenbach \& Gorti 2009, Ercolano \& Owen 2010) and the [S II] lines at 6731 and $6716 \AA$ (Ercolano \& Owen 2010).

We will now analyze our mid-infrared and optical spectra focusing on gas lines that could trace the ionized and neutral components of the disk wind.

\section{New mid-infrared observations and data reduction}

Our first VLT/VISIR observations have shown that the [Ne II] $12.81 \mu \mathrm{m}$ emission from TW Hya traces unbound gas moving toward the observer, likely in a photoevaporative

disk wind (Pascucci \& Sterzik 2009). We performed additional long-slit high-resolution spectroscopy with VISIR (Lagage et al. 2004) on 23-24 February 2010 attempting to detect the photoevaporative flow at a resolution better than the instrument resolving power ( 400 mas or $20 \mathrm{AU}$ at the distance of TW Hya). To reach this goal we have applied the so-called spectro-astrometric technique (e.g., Whelan \& Garcia 2008) and acquired spectra at four different slit orientations (position angle $\mathrm{PA}=0,270,333,63^{\circ}$ ) interleaved by four antiparallel orientations, i.e. where the slit is rotated by $180^{\circ}$ (position angle $\mathrm{PA}=180,90$, 153, $243^{\circ}$ ), see Table 1. PAs $0^{\circ}$ and $180^{\circ}$ correspond to N-S slit orientations on the sky, $270^{\circ}$ and $90^{\circ}$ to $\mathrm{W}$-E while the other two couples of PAs were chosen to cover the PA of the disk of TW Hya (i.e. the angle between $\mathrm{N}$ and the major axis of the disk measured $\mathrm{E}$ of N, see Table 2) and a direction perpendicular to it. As in past observations, we used a slit width of 0 ". 4 which provides a resolution of $\mathrm{R} \sim 30,000$ or $\sim 10 \mathrm{~km} / \mathrm{s}$ in velocity scale at $\sim 12.8 \mu \mathrm{m}$ (Käufl 2006; Pascucci \& Sterzik 2009). 
Before each spectroscopic integration we acquired TW Hya with the PAH2-NEII filter to accurately position the source in the slit. Fig. 11 shows the difference in pixels between the location of the target and the slit center in cross-dispersion direction, i.e. perpendicular to the slit length. The FWHM of the slit ( $\sim 3$ pixels) is also overplotted. Except for $\mathrm{PA}=63^{\circ}$, TW Hya was positioned within half of the slit FWHM from the slit center. We note, however, that for this specific PA the emission is also the faintest, hence the error in the source location is the largest (dashed line in Fig. 1). The source faintness could be due to flux loss caused by the imperfect centering.

We applied the standard chopping/nodding technique with a throw of $8^{\prime \prime}$ along the slit to suppress the mid-infrared background. Based on our previous experience, we set the on-source exposure time to $1 \mathrm{~h}$ for each slit orientation. For all PAs in the first night and for the first PA in the second night we also observed a standard star immediately before or after the TW Hya spectroscopic exposure and with the same slit PA to correct the spectra for telluric absorption and to obtain an absolute flux calibration. A summary of the observations is presented in Table 1 .

Each spectroscopic exposure has been reduced as described in Pascucci \& Sterzik (2009) using the VISIR pipeline version 3.2.2 (Lundin 2008). In brief, images are first corrected by fluctuations in the background using the off-source chop and nod exposures. In parallel, a reference frame of the infrared background is also created. Next, images are corrected for the optical distortion, and are shifted and added to form a final combined image from which the spectrum is extracted following the optimal extraction method by Horne (1986). Finally, the spectrum is wavelength calibrated by cross correlating the background frame spectrum to a synthetic model spectrum of the atmosphere in the observed wavelength range (note that the resulting wavelengths are in vacuum).

To remove telluric features we have used ATRAN model atmospheres for different 
elevations and amounts of precipitable water as follows. We normalized each spectrum of TW Hya to the continuum, such that the continuum is 1, and scaled the model atmospheres to minimize (in a $\chi^{2}$ statistics) the difference between the observations and the model in regions where there are strong atmospheric absorption features (the strongest one is from $\mathrm{CO}_{2}$ around $\left.12.812 \mu \mathrm{m}\right)$. During the minimization we took into account the flux uncertainty at each wavelength and also allowed for a small shift of \pm 1 pixel in wavelength to find the best scaled/shifted atmospheric model that matches the continuum of TW Hya. Since in the first night we also acquired standard stars immediately before or after each PA of the TW Hya spectra, we could verify that the approach described above gives identical results (within the flux uncertainties) to the more traditional approach of dividing each science spectrum by that of a flux calibrated standard star spectrum. Fig. 2 presents the five TW Hya spectra that could be flux calibrated while Table 3 summarizes the main properties of the observed [Ne II] lines for all PAs.

\subsection{Accuracy of the VISIR wavelength solution}

Because one of our goals is to measure small shifts in the peak of the [Ne II] line, we performed additional tests to verify the instrument wavelength solution and investigate the relative and absolute wavelength accuracy of VISIR in the high-resolution long-slit mode. First of all, we reduced and analyzed 8 additional archival spectra from 3 different infrared-bright K4-6 III standard stars (HD 136422, HD 139127, HD 149447) observed in

the same setting as TW Hya. After applying the proper heliocentric correction and shifting the spectra in the stellocentric frame, we compared the observed peak position of two photospheric absorption lines to those predicted by the MARCS model atmosphere of a K5 III star (HR 6705; the spectrum was kindly provided by L. Decin). While differences in velocities are confined within $\pm 2 \mathrm{~km} / \mathrm{s}$ it is apparent that one of the lines is typically 
redshifted while the other is typically blueshifted. To investigate whether there could be an issue in the wavelength dispersion solution and whether errors could be larger at other wavelengths, we turned to spectra of Titan which were acquired with the same setting as TW Hya (VLT/VISIR PID 083.C-0883). Titan spectra present many unresolved lines from $\mathrm{C}_{2} \mathrm{H}_{2}$ and $\mathrm{C}_{2} \mathrm{H}_{6}$ molecules in this setting. In case of large drifts in wavelength, we might see the FWHM of unresolved lines to change across the spectrum. We measured the FWHMs of 7 unresolved emission lines in two spectra of Titan covering the full VISIR wavelength range (3 before and 4 after the [Ne II] line in TW Hya) and found no such trend, rather we report a narrow range of FWHMs with a mean value of $10 \mathrm{~km} / \mathrm{s}$, the spectral resolution of VISIR in this setting. In addition, we compared the location of Titan lines with the rest wavelengths reported in the HITRAN database (Rothman \& Gordon 2009). In the setting covered with VISIR there are seven transitions from the $\mathrm{C}_{2} \mathrm{H}_{6}$ molecule, 2 of which are single and can be used to measure the relative centroids to other lines through the spectrum. Fig. 3 shows that the relative uncertainty between the lines is $1 \mathrm{~km} / \mathrm{s}$, one-tenth of the spectral resolution of VISIR in this mode. However, as hinted by the test on the standard stars and as we will see on the TW Hya data (Sect. 4), the absolute accuracy reachable with VISIR is likely a factor of 2 higher $(2 \mathrm{~km} / \mathrm{s})$, due to uncertainties in placing the source in the center of the slit.

\section{Constraints on the wind structure from ionized and neutral species}

In the following we analyze our new mid-infrared high-resolution spectra with special emphasis on characterzing the [Ne II] peak velocities and the overall line profile. We also re-analyze already published high-resolution optical spectra of TW Hya to search for additional transitions from neutral and ionized species that may trace the photoevaporative flow. The optical spectra were obtained between 1998 and 2000 with the FEROS 
echelle spectrograph on the $1.5 \mathrm{~m}$ ESO telescope on La Silla at a resolution of $\sim 6 \mathrm{~km} / \mathrm{s}$ ( $R \sim 48,000)$. Alencar \& Batalha (2002) presented an extensive analysis of the optical variability of TW Hya based on this dataset. We refer to their paper for details on the data reduction.

\section{1. [Ne II] line fluxes and peak velocities}

Spitzer/IRS spectra of TW Hya taken in three different years show variations of up to $\sim 30 \%$ in the continuum emission, as well as in the [Ne II] flux and equivalent width (Najita et al. 2010). These differences may arise from pointing errors and/or true source variability. The VISIR spectra we acquired in this observational campaign provide additional epochs to further investigate continuum and line variability on the timescale of years as well as on the much shorter timescale of hours.

For each VISIR spectrum we compute the location of [Ne II] peak emission in the stellocentric frame (after assuming a stellar radial velocity of $12.2 \pm 0.5 \mathrm{~km} / \mathrm{s}$, Weintraub et al. 2000), the EW, and when standard stars were acquired close in time to the TW Hya spectroscopic exposures, also the line flux and continuum near the line (essentially for each PA in the first night and for the first PA in the second night). Peak positions and [Ne II] line fluxes are calculated from gaussian fits to the observed line profiles, while EWs are computed by integrating the line flux within $\pm 4 \sigma$ of the fitted gaussian. Table 3 summarizes the main results and Fig. 2 shows line profiles for the five flux calibrated spectra.

To test the hypothesis of variations due to calibration uncertainties, we search for correlations between the main properties of the [Ne II] line and the adjacent continuum with the airmass and the location of the source in the slit. We find an anti-correlation 
between the line flux and continuum and the source airmass (lower airmass $\rightarrow$ higher flux and continuum, see Fig. 4) suggesting that most variations in these quantities are due to flux calibration uncertainties. We do not find any correlation between these quantities and the location of the source in the slit. However, we note that the displacement of the source with respect of the center of the slit might be the cause for the lowest EW and the largest blueshift in the $[\mathrm{Ne}$ II $]$ line for $\mathrm{PA}=63^{\circ}$ (see Fig. 1 and Fig. 4). Unfortunately, we do not have a standard star to flux calibrate the TW Hya spectrum at this PA but our expectation is of a reduced line flux and continuum. We also note that the highest [Ne II] flux we measure (for $\mathrm{PA}=180^{\circ}$, smallest airmass) is as high as that measured by Spitzer/IRS within the estimated errorbars. This demonstrates that the small VISIR slit recovers all the [Ne II] emission from the disk of TW Hya implying that the emission is confined within $\pm 10 \mathrm{AU}$ from the star.

[Ne II] line EWs are less affected by differences in the airmass: for the 5 exposures at airmass $<1.3$ (excluding $\mathrm{PA}=63^{\circ}$ ) the mean $\mathrm{EW}$ is $46 \pm 2 \AA$. This value is slightly larger than the [Ne II] EW from our 2008 high-resolution spectrum (Pascucci \& Sterzik 2009), the same as the 2006 EW measured with Spitzer/IRS (Najita et al. 2010), and smaller by about $20 \AA$ than the 2004 and 2008 EWs measured with Spitzer/IRS and the 2007 EW measured with Gemini/Michelle by Herczeg et al. (2007). Although the sampling is sparse, the [Ne II] EW essentially oscillates around two values $(\sim 45$ and $65 \AA)$ which suggests intrinsic variability in the $[\mathrm{Ne}$ II] emission or nearby continuum on a timescale of $\sim 1-2$ years.

We also confirm the blueshift in the [Ne II] line with respect to the radial velocity of TW Hya (see Fig. 4 second panel). The mean blueshift is $-5 \pm 1 \mathrm{~km} / \mathrm{s}$ in agreement with our previously reported blueshift (Pascucci \& Sterzik 2009). The peak position of the [Ne II] line is not correlated with the airmass at which the source was observed, nor with the location of the source in the slit (but see previous note for $\mathrm{PA}=63^{\circ}$ ). The $\mathrm{FWHM}$ of 
the $[\mathrm{Ne}$ II] line presents the least variations with PA, is not correlated with the EW, peak position, nor with the airmass: the mean value and standard deviation for the FWHM are $16.3 \pm 0.9 \mathrm{~km} / \mathrm{s}$, or an intrinsic $\mathrm{FWHM}$ of $12.9 \pm 0.9 \mathrm{~km} / \mathrm{s}$ after deconvolution with the instrumental FWHM of VISIR of $10 \mathrm{~km} / \mathrm{s}$.

\subsection{Forbidden and semi-permitted optical lines}

We search the FEROS optical spectra for all the lines predicted by Font et al. (2004), Hollenbach \& Gorti (2009), and Ercolano \& Owen (2010) to have luminosities of a few $10^{-7} \mathrm{~L}_{\odot}$ or higher, thus comprising forbidden and semi-permitted transitions from OI, OII, MgI, NII, and SII. We report equivalent widths and upper limits in Table 4 for representative transitions. To search for weak lines and properly measure EWs we had to correct for the numerous photospheric absorption lines present in the optical spectra of TW Hya. We followed the approach described by Alencar \& Batalha (2002) and divided the continuum normalized spectra of TW Hya with the artificially veiled and continuum normalized spectrum of GJ 1172, a K7 V star with similar v sini as TW Hya (see also Hartigan et al. 1989 for the procedure and definition of veiling). In addition, we combine all observed spectra in a median high signal-to-noise spectrum normalized to the continuum level.

Optical spectra from $\mathrm{T}$ Tauri stars are known to have strong forbidden emission lines from [OI] at 6300, 6364, and $5577 \AA$ (Hartigan et al. 1995). TW Hya is no exception and these transitions are easily identified in all individual spectra. The strongest of all is the [OI] line at $6300 \AA$ with a median EW of $0.47 \AA$, which was found not to vary significantly with time (Alencar \& Batalha 2002). Following Hartigan et al. (1995) and assuming a mean $\mathrm{R}$ magnitude of 10.2 , we convert this EW into a line luminosity of $10^{-5} \mathrm{~L}_{\odot}$. The optical spectrum of TW Hya is known to vary, the most detailed V magnitude monitoring 
reports night-to-night variations of $\Delta V \simeq 0.35$ (seasonal changes have smaller amplitudes, Rucinski et al. 2008). The same variations in $\mathrm{R}$ convert into a [OI] luminosity range of $0.8-1.6 \times 10^{-5} \mathrm{~L}_{\odot}$. The $6364 \AA[\mathrm{OI}]$ line is a factor of $\sim 3$ weaker than the [OI] $6300 \AA$, as expected from the ratio of the Einstein coefficients, while the $5577 \AA$ [OI] line is a factor of $\sim 7$ weaker on average (see Table 4). These lines are also spectrally resolved with FEROS, the mean (and median) FWHM of the [O I] $6300 \AA$ is $11.5 \pm 0.4 \mathrm{~km} / \mathrm{s}$ or an intrinsic line width of $\sim 10 \mathrm{~km} / \mathrm{s}$ after deconvolution with the $6 \mathrm{~km} / \mathrm{s}$ instrumental FWHM. The other weaker [OI] lines have similar FWHMs. As already reported in Alencar \& Batalha (2002) the [O I] $6300 \AA$ line presents only a narrow component, is centered at the stellar rest frame, and there is no indication of a blueshifted high-velocity component from jets/outflows. We use 40 spectra from Alencar \& Batalha (2002) to further investigate whether there is a blueshift of just a few $\mathrm{km} / \mathrm{s}$ in the [O I] peak emission 2 . To do that we rely not only on the absolute wavelength calibration performed previously but also compare the location of the [O I] $6300 \AA$ line with respect to other known photospheric lines. Fig. 5 shows the distribution of velocities for the [O I] $6300 \AA$ line in the stellocentric frame measured via the photospheric CaI $6439 \AA$ line (black dashed line). This histogram peaks at negative velocities but the median difference velocity is only $-0.1 \mathrm{~km} / \mathrm{s}$ with a standard deviation as large as $0.15 \mathrm{~km} / \mathrm{s}$. While this tiny blueshift might indicate a flow, we show in the same figure that it is almost certainly related to small differences in the dispersion solution

\footnotetext{
${ }^{2}$ We excluded the 10 spectra obtained by Alencar \& Batalha (2002) in December 1998 because the terrestrial night-sky emission line at [OI] $6300 \AA$ falls at $+12 \mathrm{~km} / \mathrm{s}$ in the stellocentric frame thus introducing a clear bump on the red side of the [O I] line from TW Hya. For the other 40 spectra obtained in April and February the terrestrial [O I] line is blueshifted by $23 \mathrm{~km} / \mathrm{s}$ and is within $1 \mathrm{~km} / \mathrm{s}$ from the [O I] line of TW Hya respectively. Thus, the [O I] line profiles from TW Hya are not affected by the night-sky emission in these 40 spectra
} 
between the two different echelle orders in which the [O I] $6300 \AA$ and the CaI $6363 \AA$ fall. To demonstrate this we overplot the difference between the [O I] $6300 \AA$ and the [O I] $6364 \AA$ which falls in the same order as the CaI photospheric line and should trace the same disk emission as the [O I] $6300 \AA$ line. Fig. 5 clearly shows that the two velocity distributions are identical, the median difference between the [O I] $6300 \AA$ and the [O I] $6364 \AA$ being $-0.1 \mathrm{~km} / \mathrm{s}$ and standard deviation being $0.16 \mathrm{~km} / \mathrm{s}$. These tests demonstrate that the [OI] optical lines are not blueshifted.

In addition to the oxygen lines, we also detect the [S II] line at $4069 \AA$ and the $\mathrm{Mg} \mathrm{I}]$ line at $4571 \AA$ with luminosities that are about $\sim 10$ times lower than the [OI] $6300 \AA$ luminosity (Table 4). The $[\mathrm{S} \mathrm{II}]$ transition at $4076 \AA$ comes from very similar upper energies $(\sim 3 \mathrm{eV})$ as the $4069 \AA$ transition but the Einstein coefficient is 2.5 times smaller, consistent with our non-detection and $3 \sigma$ upper limit. The [S II] at $4069 \AA$ and the $\mathrm{Mg} \mathrm{I}]$ line at $4571 \AA$ peak at the stellar radial velocity as the [O I] lines. Their luminosities are similar to those predicted by the X-EUV primordial disk model with $\log (\operatorname{Lx})=29.3$ (Ercolano \& Owen 2010). However, we note that this Xray luminosity is low for TW Hya, about an order of magnitude lower than what has been estimated by e.g. Kastner et al. (1999). In the framework of photoevaporative disk models, the most puzzling result is the non-detection of the other two $[\mathrm{S} \mathrm{II}]$ transitions at 6716 and $6731 \AA$ with stringent upper limits pointing to fluxes $~ 50$ times lower than the [O I] $6300 \AA$ line. As mentioned in Sect. 2, both EUV and Xray irradiated disks should produce a strong [S II] doublet for gas at low density and flowing, similar in strength or just a factor of a few weaker than the [OI] $6300 \AA$ line. While the [SII] 6716/6731 doublet should trace more extended low density gas than other forbidden lines due to its much lower critical density 3 , it is unlikely that the FEROS spectra

\footnotetext{
${ }^{3}$ according to EUV models the radial extension is a factor of 2 greater than the [Ne II] emission (out to about $\pm 20 \mathrm{AU}$ from the star) and less concentrated to the center
} 
lost most of the [SII] 6716/6731 flux since the fiber diameter projected on the sky is $2.7^{\prime \prime}$ and covers radii out to $\sim 70 \mathrm{AU}$ at the distance of TW Hya. Thus, the non-detection of these transitions points to specific conditions in the disk emitting region, specifically to dense gas, as we shall discuss later.

Finally, the centrally peaked profiles and the FWHM of the optical lines can help constrain the extension of the emitting region. The [OI] $6300 \AA$ line has an intrinsic width of $10 \mathrm{~km} / \mathrm{s}$, the $[\mathrm{SII}]$ and $\mathrm{MgI}]$ lines are similarly narrow $\sim 10-12 \mathrm{~km} / \mathrm{s}$, hence likely trace similar disk radii. For a given ring of gas in Keplerian rotation extending out to a radius

$r_{\mathrm{o}}$ from the star the absence of a double peak implies: $2 \sqrt{\left(\frac{G M_{\star}}{r_{\mathrm{O}}}\right)} \sin (i)<\Delta v_{\mathrm{FEROS}}$ or $r_{\mathrm{o}}>0.4-1 \mathrm{AU}$ for the mass and disk inclinations reported for TW Hya (Table 2). Again for gas in Keplerian rotation, the inner ring radius $r_{\mathrm{i}}$ determines the maximum velocity in the line profile. Because the observed [OI] $6300 \AA$ profiles extend out to $\pm 10 \mathrm{~km} / \mathrm{s}$ (see Sect. 4.3) the inner radius of the gas ring should be $<0.2 \mathrm{AU}$ and can be as close to the star as $0.06 \mathrm{AU}$ for a $4^{\circ}$ disk inclination. This inner disk gas is likely not photoevaporating. However, if most of the emission is extended beyond about $1 \mathrm{AU}$ and gas is photoevaporating, detailed disk models are necessary to compute line profiles and estimate the extension of the emitting region (see Sect. 5.2).

\subsection{Comparison of $[\mathrm{Ne} \mathrm{II}]$ and $\left[\begin{array}{ll}\mathrm{O} & \mathrm{I}] \text { line profiles }\end{array}\right.$}

Our first high-resolution VISIR spectrum of TW Hya showed a hint for an asymmetry in the [Ne II] line profile (see Fig. 4 from Pascucci \& Sterzik 2009), with possibly more emission on the blue side than on the red side of the line. A flux enhancement on the blue side was also pointed out by Herczeg et al. (2007) in their lower signal-to-noise spectrum. Such an enhancement is expected for face-on disks with an optically thick midplane blocking the view of the redshifted outflowing gas (Alexander 2008; Ercolano \& Owen 2010). We use 
the 8 additional spectroscopic exposures acquired in this observational campaign to further investigate the [Ne II] $12.81 \mu \mathrm{m}$ profile. To check for asymmetries we apply the following method: We produce for each line a mirror spectrum (in velocity scale), then we apply small shifts in velocities to the mirror spectrum such that the upper half of the lines matches best, finally we subtract the shifted-mirror and original spectra. This method is especially sensitive to detect any blue or red excess emission in the wings of the line compared to its core emission and is more suited to lines that are poorly sampled in velocity than the line bisector method (e.g., Gray 1980).

Fig. 6] summarizes our findings. Individual line profiles as well as the median of line profiles (thick black line) show an excess emission on the blue side of the [Ne II] line peaking around $-20 \mathrm{~km} / \mathrm{s}$ and a typical shift of the peak centroid of $-5 \mathrm{~km} / 4$. The blue excess is also visible as decreased emission on the red side of the line at $\sim 10 \mathrm{~km} / \mathrm{s}$ (see upper and lower panels of Fig. 6). We can confirm that this asymmetry is intrinsic to the source emission and not an instrumental effect by applying the same method to spectrally unresolved emission lines from Titan (see Fig. 7).

We then apply the same method of mirrored spectra to simulated [Ne II] line profiles from photoevaporative disk winds. As explained in Sect. 2, such profiles are available for the EUV-only (Alexander 2008) and the X-EUV (Ercolano \& Owen 2010) models. These models use input parameters such as stellar mass, Xray and EUV luminosities that are similar to measured values for the TW Hya system but were not specifically tuened for

\footnotetext{
${ }^{4}$ The two most different curves are for $\mathrm{PA}=270$ and $333^{\circ}$. The line profile from the first PA shows decreased emission (one pixel) at $\sim 20 \mathrm{~km} / \mathrm{s}$ and a positive (one pixel) emission at $\sim$ $30 \mathrm{~km} / \mathrm{s}$. The subtraction of the mirror-shifted profile (having reduced emission at $-30 \mathrm{~km} / \mathrm{s}$ and excess at $20 \mathrm{~km} / \mathrm{s}$ ) generates the pronounced peak at $-30 \mathrm{~km} / \mathrm{s}$ in the original-mirror profile. The line for $\mathrm{PA}=333^{\circ}$ is consistent with a symmetric profile.
} 
TW Hya (in the case of the X-EUV we use the models with $\log \left(\mathrm{L}_{\mathrm{X}}\right)=30.3$ which are appropriate for TW Hya). In all cases the disk midplane is optically thick thus introducing an asymmetry in the line profile which is, as expected, more pronounced the closest the disk is to the face-on orientation (see Fig. 9). Applying the KS test to the difference curves shows that the XEUV $10^{\circ}$ disk inclination model has just a $6 \%$ probability that the observed and model profiles are drawn from the same parent population hinting to a difference. All other models (EUV with $i=3,10^{\circ}$ and XEUV with $i=3^{\circ}$ ) have probabilities as high as $40 \%$ meaning that they are all equally good matches to the data. The important result from this analysis is that the [Ne II] emission must arise from a region in the disk that is optically thick to the redshifted outflowing gas. We shall discuss in Sect. 5 what this implies for the extension of the ionized wind component of the photoveporative flow.

Finally, we also apply the method of mirrored spectra to the strongest of the optical lines, the $[\mathrm{OI}] 6300 \AA$ line, to check for asymmetries in the line profile. Fig. 8 shows that, unlike the $[\mathrm{Ne} \mathrm{II}]$ line, the $[\mathrm{OI}]$ profile is symmetric and the line is centered at the stellar velocity. As we will discuss in more detail in Sect. 5, this profile could result from: i) pure Keplerian motion of gas bound to the disk (i.e. the [O I] is not tracing the neutral component of the wind, it is not flowing); or ii) a photoevaporative flow from a disk region that is optically thin to the redshifted outflowing gas. Examples of symmetric lines from photoevaporative flows are presented in Ercolano \& Owen (2010) for disks with inner holes in their dust and gas distribution and emission confined within the hole. Because most of the opacity is expected to be from dust (see Sect. 5) and dust extinction decreases with wavelength, our line profile analysis demonstrates that if scenario ii) applies the neutral [O I] gas does not trace the same radii as the [Ne II] emitting gas. 


\subsection{Spectroastrometric signal of a photoevaporative wind}

We attempted for the first time spectroastrometry in the mid-infrared to improve upon the spatial resolution achievable with the VLT/VISIR and resolve the innermost region of the photoevaporative wind. Spectroastrometry relies on the fact that the measurement of the centroid of a flux distribution can be determined to much better than a pixel precision depending on the signal-to-noise of the observations. In the case of VISIR one pixel corresponds to 127 mas meaning that we could investigate the [Ne II] emission at scales better than $6 \mathrm{AU}$ at the distance of TW Hya. Asymmetries in the emission at a specific wavelength appear as shifts in the centroid of the flux distribution. Using different slit PAs enables to determine the orientations at which asymmetries are stronger and thus help constraining the distribution of the source emission.

To retrieve the spectroastrometric signal from our VLT/VISIR data we extract spatial profiles in each velocity channel and measure the spatial centroid of the [Ne II] emission in cross-dispersion direction with respect to the centroid of the emission in the continuum. The continuum is measured as the mean of the continuum centroids on the red and on the blue side of the [Ne II] line. Because of the low S/N on the continuum we summed up the spatial profiles over more than 10 pixels in velocity, we have tested that our results do not vary if we change the size of the velocity bins or the location where we measure the continuum. To determine the centroid of the spatial profiles we have tested Gaussian and Lorentzian profiles as well as the weighted mean over the FWHM of VISIR in spatial direction as proposed by Pontoppidan et al. (2008). We find that fitting the emission with Lorentzian and Gaussian profiles provide the same spectroastrometric signal and a more robust centroid than the weighted mean approach. As uncertainty on the centroid we take the inverse of the $\mathrm{S} / \mathrm{N}$ for each spatial profile where the noise is measured as the standard deviation of the continuum outside the profile. This error reflects that profiles extracted 
at velocities sampling the wings of the line have lower $\mathrm{S} / \mathrm{N}$, hence the centroid is less well determined. The centroid is also diluted by the continuum flux at each velocity to take into account the different continuum-to-line ratio moving from the core to the wing of the line (e.g., Whelan \& Garcia 2008). Fig. 10 shows the centroids in pixels versus velocities in the stellocentric frame for the parallel and anti-parallel slit orientations separately. This way we can better search for any possible instrumental effects on the centroids because such effects would appear as shifts of the same sign and intensity in the couple of parallel and anti-parallel slits (Bailey 1998). The plot does not show any strong similar effect but small amplitude modulations across the [Ne II] line and nearby continuum. As we will see next the centroids are measured to an accuracy of a tenth of a pixel close to the [Ne II] peak emission and typically to half of a pixel in the wings where the $\mathrm{S} / \mathrm{N}$ of the flux distribution in cross-dispersion direction is low 5 .

\subsubsection{Comparison between observations and EUV-model predictions}

The simple EUV-only disk model is computationally fast enough that we can create channel maps for the [Ne II] emission and compute spectroastrometric curves for the slit orientations used in this observational campaign. The model is essentially identical to the EUV-only model used by Font et al. (2004) and Alexander (2008) with a radially continuous gas distribution. The only differences between our hydrodynamic model and that of Alexander (2008) are the resolution of the model grid, and the location of the lower boundary. As we wish to generate spatially-resolved emission maps, it is necessary

\footnotetext{
${ }^{5}$ We note that the low accuracy in the centroid for $\mathrm{PA}=63^{\circ}$ is due to the lower flux in the line and continuum likely caused by the fact that the source was not well centered in the slit for this PA, see Fig. 1
} 
to use higher spatial resolution than that used by Alexander (2008). In addition, we now assume that the underlying disk has a finite thickness, and place the base of the ionized wind at $z / r \simeq 0.15$ (e.g., Hollenbach et al. 1994). This has only a small effect on the predicted line profile, reducing the peak blue-shift by approximately $1 \mathrm{~km} / \mathrm{s}$. Our polar $[(r, \theta)]$ computational grid covers the range $r=\left[0.03 r_{\mathrm{g}}, 10.0 r_{\mathrm{g}}\right], \theta=\left[0,75^{\circ}\right]$, with grid cells that are logarithmically spaced in $r$ and linearly spaced in $\theta$. We use $N_{r}=1113$ cells in the radial direction and $N_{\theta}=250$ cells in the polar direction. When the grid is expanded to three dimensions (by assuming reflective symmetry about the disk mid-plane and azimuthal symmetry around the polar axis), the resulting grid has $1113 \times 600 \times 1200$ cells in the $r, \theta$ and $\phi$ directions respectively.

We compute the line emission from each grid cell in the same manner as Alexander (2008). However, following the results of Hollenbach \& Gorti (2009) we adopt an ionization fraction for Ne II of 0.75 (rather than the somewhat arbitrary value of 0.33 used previously). For comparison to the TW Hya system we adopt a stellar mass of $M_{*}=0.7 \mathrm{M}_{\odot}$ (thus $r_{\mathrm{g}}=6.2 \mathrm{AU}$ ) and a disk inclination of $i=4^{\circ}$ (see Table 2 for references). We set the ionizing flux $\Phi$ by matching the predicted line luminosity to the observed value of $3.1 \times 10^{-6} \mathrm{~L}_{\odot}$ : we find that $\Phi=7.5 \times 10^{40}$ ionizing photons per second matches the observed line flux to $\sim 1 \%$ accuracy. The integrated mass-loss rate from the disk is $\sim 1.5 \times 10^{-10} \mathrm{M}_{\odot}$. We create an integrated line profile (identical to that of Alexander 2008) by summing the contributions from every cell on the grid, and also generate spatially resolved emission maps by de-projecting the three-dimensional grid on to the sky plane. This is done at each (spectral) velocity to generate a three-dimensional data-cube. The model data cube has $201 \times 201$ pixels which are $0.255 \mathrm{AU}$ on a side (equivalent to 5 mas at the 51 pc distance of TW Hya), and a velocity resolution of $0.1 \mathrm{~km} / \mathrm{s}$. We model the spectral resolution of VISIR by convolving the data cube (in the velocity direction) with a Gaussian profile with half-width $\sigma=5 \mathrm{~km} / \mathrm{s}$, to give a simulated spectral resolving power of $R=30,000$. 
Fig. 11] shows two simulated images of the [Ne II] emission taken at a negative and a positive velocity, the image orientation is as in Hughes et al. (2011) when $\mathrm{N}$ is up and left is $\mathrm{E}$ in the figure. To simulate the spectroastrometric signal we apply a slit in the $\mathrm{x}$-axis equal to the VISIR slit width of 400 mas, collapse the flux in the x-axis and evaluate the centroid of the spatial profile in the y-axis after convolution with the VISIR PSF (FWHM of 300 mas) for each velocity channel. To simulate the rotation of the slit we rotate the simulated images by the corresponding angle and then apply the slit as described above. Fig. 12 shows the expected spectroastrometric signal as parallel minus anti-parallel centroids in VISIR pixels for the photoevaporative disk model (solid lines) and for the wind only, i.e. when Keplerian rotation is set artificially to zero (dashed lines). One sees that for a slit orientation close to the disk major axis $\left(\mathrm{PA}=333^{\circ}\right)$ the spectroastrometric signal is dominated by the Keplerian component of the gas, the asymmetry in the emission is small but present due to the small but non-zero disk inclination. The positive displacement at negative velocities can be understood by looking at Fig. 11, the emission at negative velocities is stronger in the direction of $\mathrm{PA}=333^{\circ}$ and slit orientations close to it. The wind component dominates the signal for the slit orientation perpendicular to the disk major axis $\left(63^{\circ}\right.$ but it is prominent also for $\left.270^{\circ}\right)$ because at that orientation the Keplerian component is close to zero. The main results from the modeling are as follows: i) the expected shift in centroid is small, about 0.2 pixels, and ii) the shift is primarily set by projection effects (disk inclination) and the Keplerian component of the gas since the wind is more symmetrically distributed around the star.

To compare model predictions with observations, we subtract the measured anti-parallel centroid positions from the parallel positions (after interpolation over the same velocity grid), see Fig. 13. For PAs 270 and $63^{\circ}$, slits oriented close to the disk minor axis, the spectroastrometric shift is less than 0.1 pixel as expected. For the other two slit orientations, sampling the disk major axis, we do not detect the S-shape profile predicted for gas in 
Keplerian rotation. We see a hint of such a profile only for $\mathrm{PA}=333^{\circ}$ negative velocities

which is however not present at the positive velocities. A smaller shift than predicted could result from a [Ne II] emission that is more symmetric than predicted, from a smaller disk inclination, and/or from [Ne II] emission more closely concentrated to the central star (in this last case, the convolution with the VISIR PSF would smear out the contrast between the bright and faint rims of the [Ne II] emission). Further observations at even higher spectral resolution and sensitivity, especially in the wings of the line, are necessary to confirm these first results and to possibly identify the small spectroastrometric shift from photoevaporating gas that is expected for slit orientations along the minor axis.

\section{Physical implications on the disk wind}

Before discussing the possible wind structure from the disk of TW Hya we wish to briefly summarize the main observational results presented in the previous Sections.

- We confirm that the [Ne II] emission line at $12.81 \mu \mathrm{m}$ is blueshifted by $\sim 5 \mathrm{~km} / \mathrm{s}$ with respect to the stellar velocity. The line is relatively narrow (FWHM $13 \mathrm{~km} / \mathrm{s}$ ) and the profile is asymmetric, with more emission on the blue than on the red side.

- None of the forbidden and semi-permitted optical lines investigated here appear blueshifted. On the contrary, these lines are centered in the stellocentric frame to within about $0.15 \mathrm{~km} / \mathrm{s}$. The line profiles are symmetric and the similar FWHMs $(\sim 10 \mathrm{~km} / \mathrm{s})$ from different transitions point to optical lines tracing gas at similar radii.

- The very small or zero spectroastrometric shifts for the observed PAs suggest that the [Ne II] emission from the photoevaporative wind is predominantly spatially symmetric. 
What are these results telling us about the structure of the photoevaporative wind and its extension?

\subsection{The unbound disk component}

The small blueshift in the [Ne II] line is a clear tell-tale sign of photoevaporating gas for a face-on configuration like that of TW Hya. Hence, we are sure that this line is probing the ionized component of the disk wind. This component could be the dominant wind component in the EUV case or a trace component of the mostly neutral wind in the Xray case. Our observations constrain the radial extension of the ionized wind component via the [Ne II] line. By recovering all flux measured with Spitzer/IRS, we demonstrate that the [Ne II] emission arises within $10 \mathrm{AU}$ from the star. We note that although the radial extension of the mass loss is very different for pure EUV and X-EUV irradiation, with Xrays producing $50 \%$ of the mass loss at tens of AU (Owen et al. 2010), the [Ne II] luminosity decreases steeply with radial distance from the star (as $\sim 1 / r$ ) hence the emission is dominated by the flux from small radii. For the specific EUV and X-EUV models discussed here $90 \%$ of the [Ne II] emission arises within $12 \mathrm{AU}$ and $15 \mathrm{AU}$ respectively, which is consistent with the $10 \mathrm{AU}$ radial extension inferred observationally.

The blue excess in the wing of the [Ne II] line adds a further constraint on the radii traced by this wind component. We have shown in Sect. 4.3 that primordial disks (no gaps)

irradiated by EUV and Xrays can reproduce the [Ne II] line profile and peak emission. Here we ask the question: how much [Ne II] emission could originate in an optically thin region and still be consistent with the observed [Ne II] profile? To simulate the [Ne II] profile from optically thin disk radii we take a gaussian centered at zero velocity and FWHM set by thermal broadening for gas at $\sim 5,000 \mathrm{~K}$ (Xray heating) or $\sim 10,000 \mathrm{~K}$ (EUV heating) and convolved with the FWHM of the VISIR spectrograph. We add as much as 10, 20, and 30\% 
of the total emission to the [Ne II] profile from a fully thick disk and compare the resulting profile with the observed one. We find that already a $10 \%$ contribution from radii optically thin to the [Ne II] emission shifts the line peak emission redward to $\sim-4 \mathrm{~km} / \mathrm{s}$, marginally consistent with our measurements, and increases the symmetry of the line. This simple approach shows that more than $80 \%$ of the [Ne II] emission should come from a disk region that is fully optically thick to the [Ne II] line. What is then the source of opacity within 10 AU from the central star? This is a relevant question for transition-type sources like TW Hya whose SEDs clearly point to opacity gaps in their disks.

First we look at the gas component and show that the [Ne II] line is optically thin and gas alone cannot be responsible for the blueshift and line asymmetry. Hollenbach \& McKee (1989) calculated that a column of $\mathrm{Ne}^{+}$of a few times $10^{18} \mathrm{~cm}^{-2}$ (or a few $10^{22} \mathrm{H}$ atoms for a solar $\mathrm{Ne} / \mathrm{H}$ abundance ratio) is necessary to produce an optical depth of 1 at the center of the [Ne II] line. This density has to be compared with $<10^{16} \mathrm{~cm}^{-2} \mathrm{Ne}^{+}$in the EUV layer and $<10^{17} \mathrm{~cm}^{-2}$ in the Xray layer (Hollenbach \& Gorti 2009; Glassgold et al. 2007), clearly not sufficient to make the line optically thick. That the line is optically thin is also clear from the observed line profile which does not show any absorption at the line center, where the optical depth is the highest.

Sub-micron dust grains are the next major source of opacity. According to the disk model of Calvet et al. (2002) the inner disk of TW Hya could have only a tiny amount (0.5 lunar masses) of micron-sized grains out to $\sim 4 \mathrm{AU}$ and thus would be optically thin at visible and even more at infrared wavelengths out to that radial distance. However, Ratzka et al. (2007) show that this model gives a factor of 2 too high mid-infrared visibilities as well as the wrong wavelength dependence of the visibility. They argue that the dust inner rim, hence the transition from thin to thick disk, must happen much closer in at about 0.5-0.8 AU. More recently, Akeson et al. (2011) also argue for the presence of optically 
thick dust within $4 \mathrm{AU}$. They propose a more complex structure with an optically thick dust ring centered at 0.5 AU, a dust gap, and the full optically thick dust disk starting at 4 AU. Our simple approach of combining [Ne II] profiles from optically thin and thick disk radii suggests that more than $80 \%$ of the [Ne II] emission should arise from disk radii where the midplane is optically thick to the [Ne II] emission. If the optically thin disk

extends out to $4 \mathrm{AU}$ as in the model of Calvet et al. (2002), then the observed [Ne II] profile cannot be reproduced by the EUV-only and X-EUV models from Alexander (2008) and Ercolano \& Owen (2010) because in these models 50\% of the [Ne II] emission arises within $4 \mathrm{AU}$. If the dust inner rim is at $\sim 1 \mathrm{AU}$, as in the Ratzka et al. (2007) model, then these models can explain the blueshift and asymmetry in the line profile since they both predict that $\sim 90 \%$ of the [Ne II] emission comes from outside 1 AU, i.e. from disk radii where the midplane would be optically thick. A different model, also consistent with our observations, is that from Gorti et al. (2011). From a detailed analysis of many gas emission lines from TW Hya and using the Calvet et al. dust distribution with a static disk model, they find that $75 \%$ of the [Ne II] emission line comes from the surface of the optically thick dust disk beyond the 4 AU gap.

\subsection{The disk component traced by optical lines}

Unlike in the case of the [Ne II] line, the forbidden and semi-permitted optical lines investigated here are not blueshifted with respect to the stellar radial velocity. The lack of blueshift could have two origins: i) the optical lines trace a wind component at disk radii where the midplane is optically thin to the redshifted outflowing gas; OR ii) the optical lines do not trace the photoevaporative flow but rather gas that is bound to the star/disk system. In reference to the [OI] optical lines, this means that we do not have an unambiguous diagnostic for the neutral wind component of the disk as we have for the 
ionized component. In the following, we discuss what other constraints we can place on the disk region traced by the optical lines and what observations could discriminate between the two scenarios presented above.

First of all, the similarity in the FWHM of the [OI] 5577, 6300, $6364 \AA$, the [SII] at $4068 \AA$, and the MgI] at $4571 \AA$ suggests that these lines trace similar disk radii. Based on the centrally peaked profiles and maximum observed velocities, we argue in Sect. 4.2 that the emission could extend as close as $0.06 \mathrm{AU}$ to the star, where the disk may be magnetospherically truncated, to and possibly beyond 1 AU. The observed [OI] 6300/5577 line ratio of $\sim 7$ as well as the non-detection of the [SII] doublet $6716 / 6730 \AA$ point to a region of high temperature and electron density. For gas in local thermal equilibrium (LTE) and optically thin emission we can write the [SII] 6716 and $4069 \AA$ ratio as:

$$
\frac{L_{4069}}{L_{6716}}=\frac{n\left({ }^{2} P\right) A_{4069} \Delta E_{4069}}{n\left({ }^{2} D\right) A_{6716} \Delta E_{6716}}
$$

$n\left({ }^{2} P\right)$ and $n\left({ }^{2} D\right)$ are the upper level populations which in LTE are linked as $n\left({ }^{2} P\right) / n\left({ }^{2} D\right) \sim$ $4 / 6 \times e^{-14,000 / T}$. To explain a line flux ratio higher than 6 we thus require gas hotter than $\sim 3,000 \mathrm{~K}$. A similar argument applied to the [OI] 6300 and $5577 \AA$ lines yields a similarly high temperature (Gorti et al. 2011). Such a high temperature can be reached within a few AU from the star in a disk atmosphere irradiated by stellar Xray but LTE requires very high electron densities $\left(n_{e} \gtrsim 10^{8} \mathrm{~cm}^{-3}\right)$ which may not be present in the photoevaporative wind (Gorti et al. 2011). We turn now to the strong [OI] lines and on the neutral component of the disk wind to investigate the two scenarios proposed at the beginning of this section.

In the first scenario, the [O I] emission is thermal and arises from the hot $(>5,000 \mathrm{~K})$ disk surface heated by stellar Xrays. Ercolano \& Owen (2010) have shown that line profiles can be symmetric even if the gas is flowing and any blueshift in the peak emission can be small, a few tenth of $\mathrm{km} / \mathrm{s}$, if most of the emission arises within the dust depleted inner 
disk 6 . For the specific case of TW Hya an inner dust hole of 2 AU would produce a blueshift in the $[\mathrm{OI}]$ line greater than $1 \mathrm{~km} / \mathrm{s}$ and slightly asymmetric profiles while a $4 \mathrm{AU}$ hole would give just a shift of $0.4 \mathrm{~km} / \mathrm{s}$ and a symmetric profile pointing to the flow traced by the [OI] emission extending well beyond $2 \mathrm{AU}$. The FWHM of the line is in both models relatively large $\sim 20 \mathrm{~km} / \mathrm{s}$, a factor of 2 larger than measured. The line broadening can be reduced if the emission in the flow is suppressed and the bound [O I] thermal component arising from within a few AU dominates the emission. This strongly points to the second scenario in which the $[\mathrm{OI}]$ lines are not tracing the neutral component of the photoevaporative wind in the disk of TW Hya but rather the bound disk component well inside the gravitational radius.

A variant to the second scenario is that proposed by Gorti et al. (2011). In this model, which is specifically for TW Hya, most of the [O I] emission is not thermal but produced from dissociation of $\mathrm{OH}$ molecules by stellar FUV photons leading to $\mathrm{O}$ atoms in electronically excited states and their subsequent decay to the ground state. Gorti et al. (2011) find that this mechanism gives a $6300 / 5577 \AA$ line ratio of $\sim 7$, very similar to the observed one. Because the [O I] emission comes from cooler gas closer to the disk midplane the emitting gas would not participate to the photoevaporative flow and hence [OI] lines would be narrower and symmetric around the stellar velocity. The [SII] line ratios are also reproduced in this model since most of the emission arises in the hot and dense gas close to the star within the dust depleted inner disk.

A way to directly discriminate between bound and unbound oxygen gas is through spectroastrometry in the $[\mathrm{OI}] 6300 \AA$ line. For gas in Keplerian rotation the

\footnotetext{
${ }^{6} \mathrm{As}$ in the case of the [Ne II] line sub-micron dust grains are the main source of opacity. For the $[\mathrm{OI}] 6300 \AA$ line the $\mathrm{H}$ column density to reach optical depth of one is as high as $2 \times 10^{25} \mathrm{~cm}^{-2}$.
} 
spectroastrometric signal will be symmetric in velocity and the strongest for a slit oriented along the disk major axis, the signal will be zero for a slit perpendicular to the major axis (see, e.g. Fig. 12). The presence of a photoevaporative wind alters the symmetric spectroastrometric signal and produces a shift in the flux distribution versus wavelength even for a slit perpendicular to the disk major axis. With a combination of good seeing $\left(\sim 1^{\prime \prime}\right)$ and high signal to-noise a few milli-arcsecond accuracy can be attained via spectroastrometry at visible wavelengths (see e.g. Takami et al. 2003). Hence, detecting asymmetries around $1 \mathrm{AU}$, which would produce shifts of $\sim 20$ mas, is well within the reach of this technique.

\subsection{On determining photoevaporation rates}

Our study of TW Hya represents the first attempt to identify and combine information from multiple gas lines at infrared and optical wavelengths that could trace the photoevaporative disk wind. Here, we summarize the observables reproduced by different disk models as applied to TW Hya and discuss how to nail down photoevaporation rates from protoplanetary disks.

The intensity of the [Ne II] line at $12.81 \mu \mathrm{m}$ can be reproduced by all models: in the EUV-only model ionized neon atoms trace the skin of the disk while in the X-EUV and XE-FUV models they trace a deeper, mostly neutral layer that participates to the flow. Any asymmetry in the line profile tells us where most of the emission arises with respect to the optically thick dust disk. A better determination of the transition from thin to thick disk could potentially validate or invalidate the EUV/Xray photoevaporation models (see Sect. 5.1). The strong [O I] line at $6300 \AA$ from TW Hya cannot arise from the EUV layer because this layer is mostly ionized and produces only weak [O I] emission. While the [O I] peak emission from several T Tauri stars in the Hartigan et al. (1995) sample presents 
a small blueshift pointing to a possible origin in the Xray wind, this is not the case for TW Hya. In addition, the small FWHM of the [O I] line also argues against most of the emission tracing the wind and rather points to bound gas close to the star. The luminosities of the [S II] at 4068 and the Mg I] at $4571 \AA$ can be reproduced by the X-EUV primordial disk model but with a stellar Xray luminosity that is 10 times weaker than that of TW Hya (Table 1 from Ercolano \& Owen 2010). The non-detection of the [S II] doublet at 6717 and $6731 \AA$ is also puzzling in the frame of both the EUV and the X-EUV model. For our results to be consistent with these models, sulfur should be depleted in the disk wind. Depletion of more refractory elements such as silicon and aluminum has been previously proposed for the gas surrounding TW Hya (e.g., Lamzin et al. 2004). Elemental abundances therefore add an additional uncertainty when comparing observed line fluxes to model calculations. Alternatively the XE-FUV model developed for TW Hya explains the non-detections of the [S II] doublet and proposes that the observed [S II] line at $4069 \AA$ arises from dense, bound gas in the inner disk (Gorti et al. 2011). Similarly to the forbidden optical lines, the CO line profiles and their spectroastrometric signal are consistent with bound gas in Keplerian rotation around TW Hya (Pontoppidan et al. 2008).

More effort should be devoted in identifying gas lines that trace the predominantly neutral winds of XE-FUV and X-EUV irradiated disks both around TW Hya as well as around other young stars. Establishing the presence of this component would point to flow rates 1-2 orders of magnitude larger than the $10^{-10} \mathrm{M}_{\odot} / \mathrm{yr}$ predicted by EUV-only photoevaporation models. In addition, attempts should be made to empirically measure the ionizing flux impinging on the surface of protoplanetary disks via for instance line flux ratios from two successive stages of ionization of a given element or from volatile elements tracing the flow. 


\section{Concluding remarks}

In the present work we have provided observational constraints to the photoevaporative wind from the disk of TW Hya. We confirm our previous finding that the [Ne II] $12.81 \mu \mathrm{m}$ line traces the ionized component of the wind from the disk of TW Hya (Pascucci \& Sterzik 2009). This would be the dominant wind component in the EUV case and a trace component in the predominantly neutral Xray wind. From the blueshift and asymmetry in the line profile, we estimate that most $(>80 \%)$ of the $[\mathrm{Ne}$ II] flux arises from disk radii where the midplane is optically thick to the emission, meaning beyond the 1 or 4 AU dust gap inferred from other observations. We note that depending on the observations/disk modeling this gap may be not completely evacuated of dust (Calvet et al. 2002; Ratzka et al. 2007; Akeson et al. 2011). We also show that the [Ne II] emission is confined within $10 \mathrm{AU}$ from the star. Our spectroastrometry in the [Ne II] line suggests that the wind flux is rather spatially symmetric around the star but the current dataset is not of quality high enough to provide additional constraints to the structure of the photoevaporative flow. We find that optical forbidden lines such as the strong [O I] emission line at $6300 \AA$ have remarkably different profiles from the [Ne II] $12.81 \mu \mathrm{m}$ line, in that they are centered at the stellar velocity and are symmetric. The only way these lines could trace the photoevaporative flow is if they arise from a disk region physically different from that traced by the [Ne II] line, specifically from within the optically thin dust gap. However, the small FWHM of the optical lines indicates that most of the gas is likely bound, which would imply gas very close to the star (inside the $\sim 1$ AU critical radius for photoevaporation) if the Keplerian velocity contributes most to the line broadening.

The above characteristics of the gaseous disk of TW Hya can be used to speculate on the origin of its transition-like SED. One possibility for creating dust gaps in circumstellar disks and still account for accretion onto the star is via dynamical clearing by forming giant 
planets (Lubow \& D'Angelo 2006). For the specific case of TW Hya, partial dust clearing is required at most out to $\sim 4 \mathrm{AU}$ from the star (Calvet et al. 2002). Recent hydrodynamic simulations by Zhu et al. (2011) suggest that one giant planet might account for this moderate-size gap (see also Gorti et al. 2011) and still result in the measured accretion rate of $10^{-9} \mathrm{M}_{\odot} / \mathrm{yr}$ (Muzerolle et al. 2000; Alencar \& Batalha 2002). Another possibility is that the gap is not opened by a planet but rather by photoevaporation and we are catching the star in the process of dispersing its disk. Recent XEUV-driven photoevaporation models predict that there is a non-negligible amount of time $\left(2-3 \times 10^{5} \mathrm{yr}\right)$ during which photoevaporation can carve a moderate-size gap, accretion onto the star is still detectable, and dust grains are cleared out under the action of dust drag (Owen et al. 2011). We would like to stress that the on-going photoevaporation from the disk of TW Hya reported here does not exclude the existence of a giant planet and does not preclude that a forming giant planet has dynamically cleared the inner disk. Infact, a giant planet might expedite the onset of substantial (and detectable) photoevaporation by depleting the inner disk surface density which reduces the stellar accretion rate and exposes the region out of the rim with direct unattenuated flux from the star.

Besides detecting a planet at a few AU from TW Hya, there are other observables that could help discriminating between a planet-induced and a photoevaporation-induced disk gap. If the putative giant planet is on an eccentric orbit, one might see variations in the dust disk structure and related dust emission on the Keplerian timescale of the planet, a few years. Regardless of the planet's eccentricity, dynamical clearing would result in a sharp transition in surface density at the outer gap edge which could be imaged with the Atacama Large Millimeter Array.

IP is pleased to acknowledge support from the National Science Foundation (NSF) through an Astronomy \& Astrophysics research grant (AST0908479). IP thanks E. 
Flaccomio for making available the VISIR spectra of Titan and K. Pontoppidan for valuable discussions. RDA acknowledges support from the Science \& Technology Facilities Council (STFC) through an Advanced Fellowship (ST/G00711X/1). SHPA acknowledges support from Fapemig and CAPES, brazilian research agencies. This research used the ALICE High Performance Computing Facility at the University of Leicester. Some resources on ALICE form part of the DiRAC Facility jointly funded by STFC and the Large Facilities Capital Fund of BIS.

Facilities: VLT (VISIR), 1.5m ESO (FEROS). 


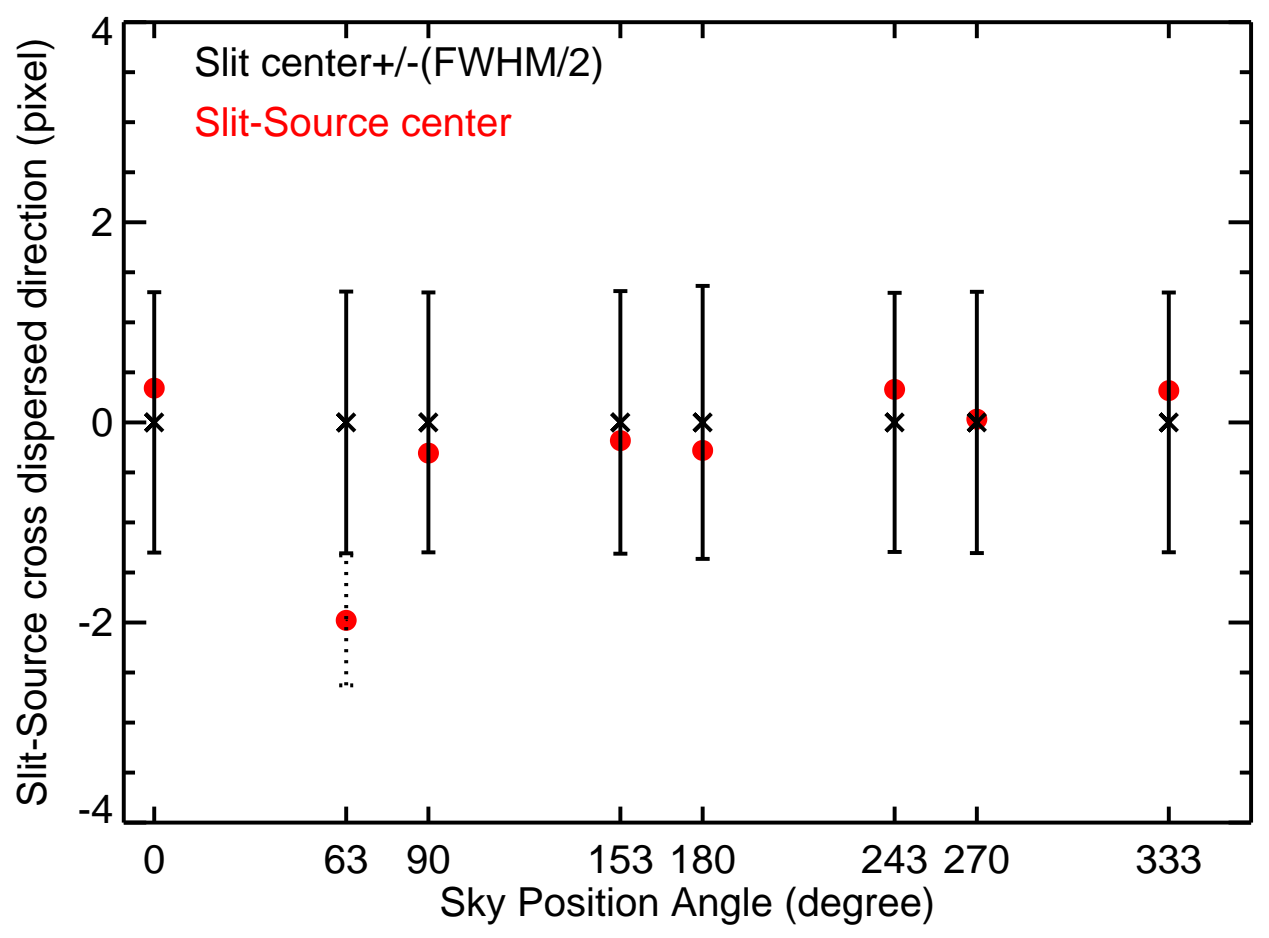

Fig. 1.- Difference in pixels between the location of TW Hya and the center of the slit before each spectroscopic integration. The x-axis gives the position angle in degrees. Vertical errorbars centered at $\mathrm{y}=0$ show the extension of the slit image (FWHM) in cross-dispersion direction. Except for $\mathrm{PA}=63^{\circ} \mathrm{TW}$ Hya was well positioned inside the slit. 


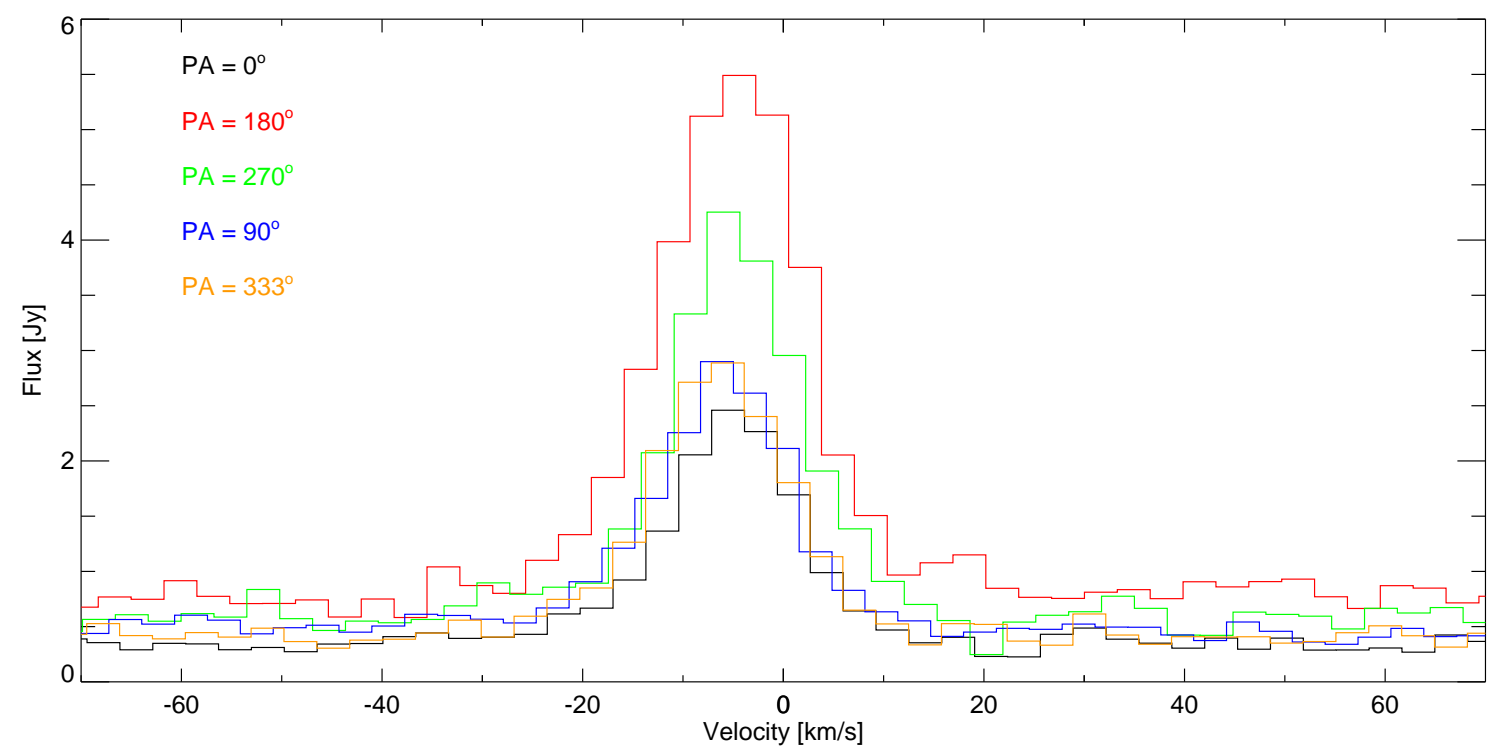

Fig. 2.- The five flux calibrated spectra of TW Hya around the [Ne II] emission line. The $\mathrm{x}$-axis gives the velocity in the stellocentric frame. 


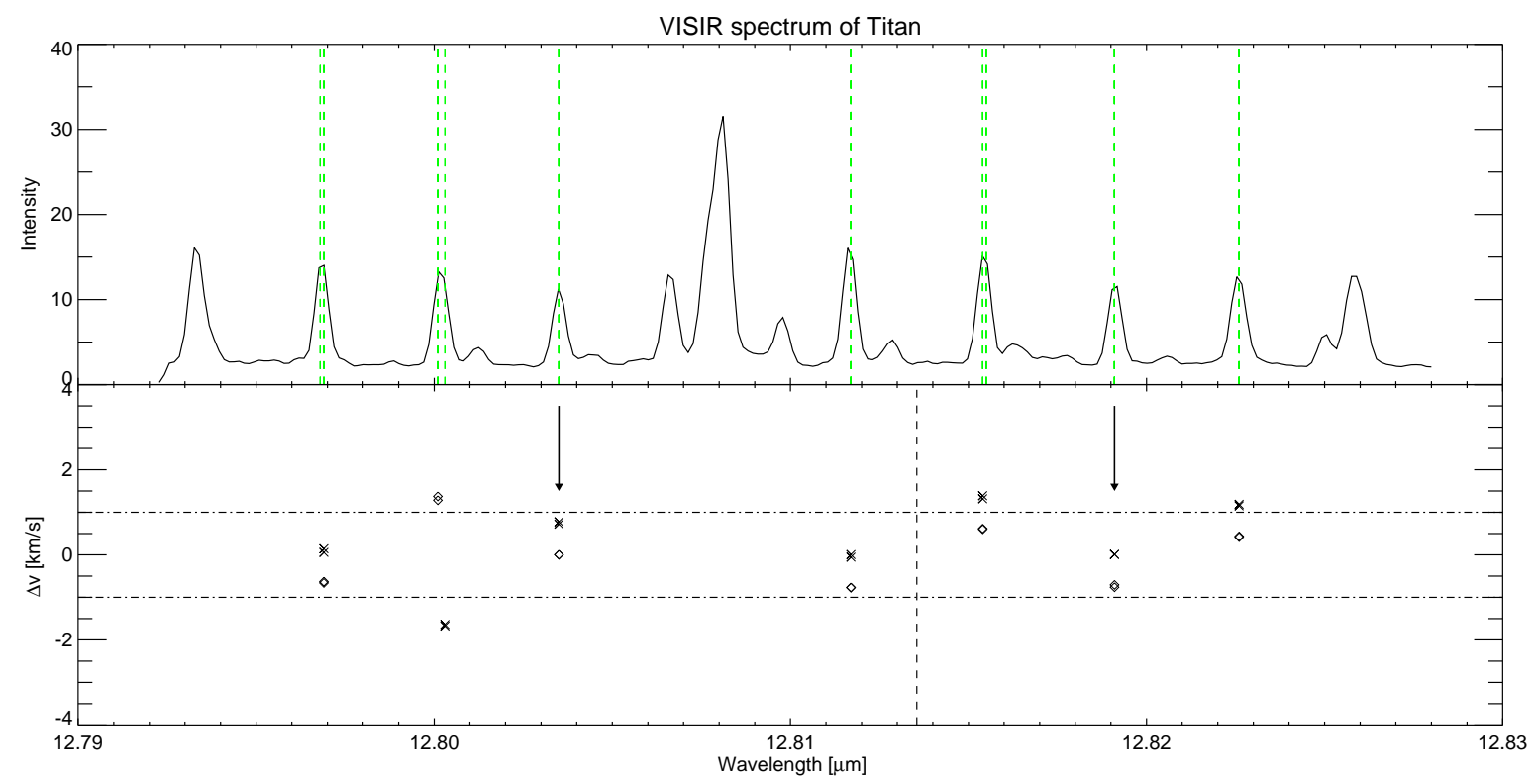

Fig. 3.- VISIR spectra of Titan demonstrating the accuracy of the dispersion solution. The upper panel marks in (green) dashed-lines the $\mathrm{C}_{2} \mathrm{H}_{2}$ transitions from the HITRAN database. This spectrum of Titan (we analyzed two spectra) has been shifted to match the single $\mathrm{C}_{2} \mathrm{H}_{2}$ line at $\sim 12.803 \mu \mathrm{m}$. The lower panel gives the velocity difference in $\mathrm{km} / \mathrm{s}$ for lines marked in the upper panel. The location of the [Ne II] line is also shown (black vertical dashed line). The two single $\mathrm{C}_{2} \mathrm{H}_{2}$ lines are marked with arrows. ' $\mathrm{X}$ ' symbols give shifts when the rest frame is computed from the single line at $\sim 12.819 \mu \mathrm{m}$, while diamonds from the single line at $\sim 12.803 \mu \mathrm{m}$. The standard deviation of the velocity difference, which gives the relative uncertainty, is $1 \mathrm{~km} / \mathrm{s}$. The absolute uncertainty is a factor of two larger (see text). 


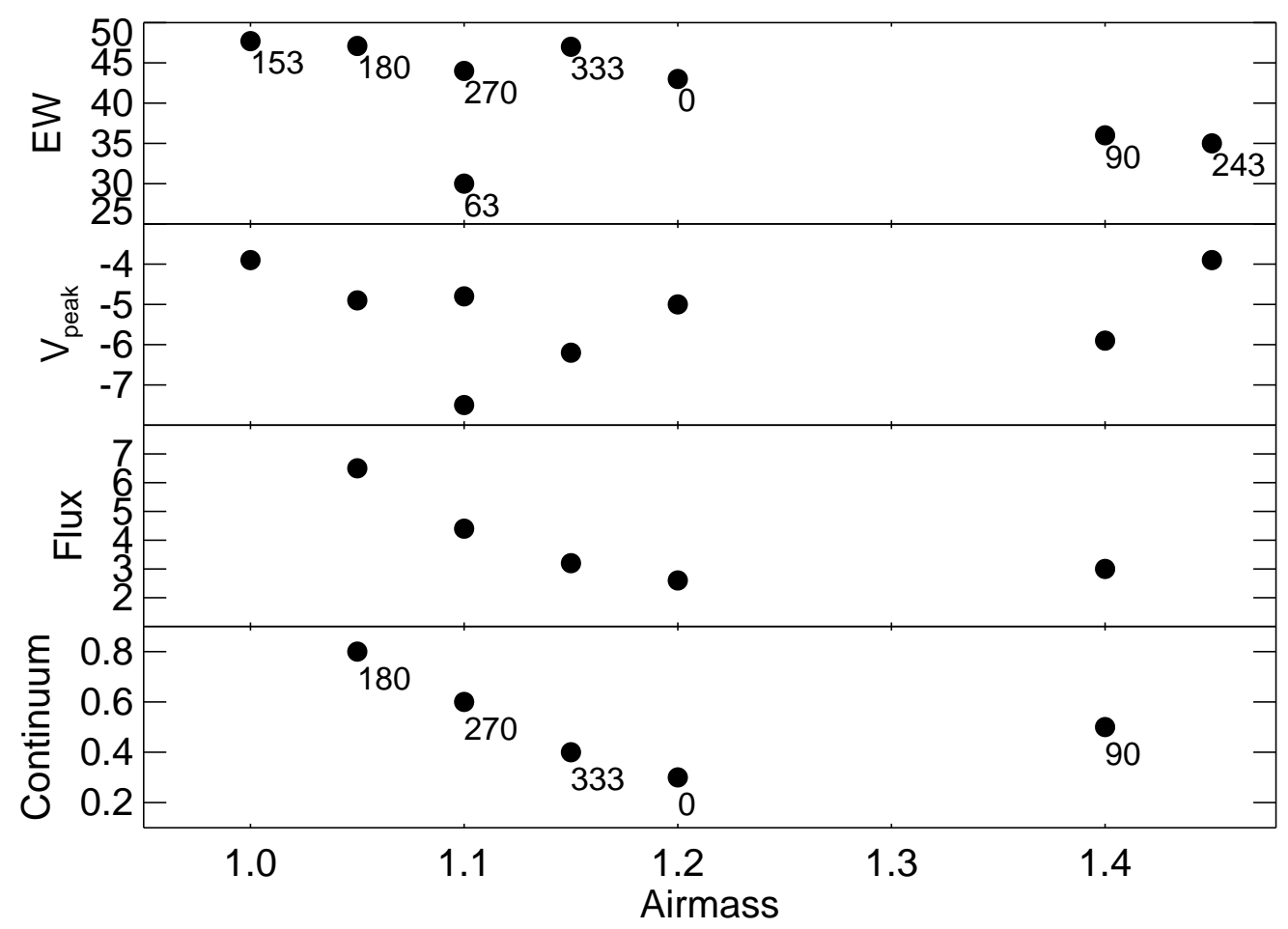

Fig. 4.- [Ne II] line EW $(\AA)$, peak centroid $(\mathrm{km} / \mathrm{s}$ in the stellocentric frame), flux $\left(\times 10^{-14} \mathrm{erg} \mathrm{cm}^{-2} \mathrm{~s}^{-1}\right)$, and adjacent continuum $(\mathrm{Jy})$ versus the airmass at which the spectroscopic observations were carried out. PAs are also overplotted. Note the anti-correlation between the [Ne II] flux and adjacent continuum with airmass suggesting that most of the observed variations in these quantities are related to flux calibration uncertainties rather than to intrinsic source variability. Also note that for $\mathrm{PA}=63^{\circ}$, which shows the lowest EW and the largest blueshift, the source was not well centered in the slit (see Fig. 1). 


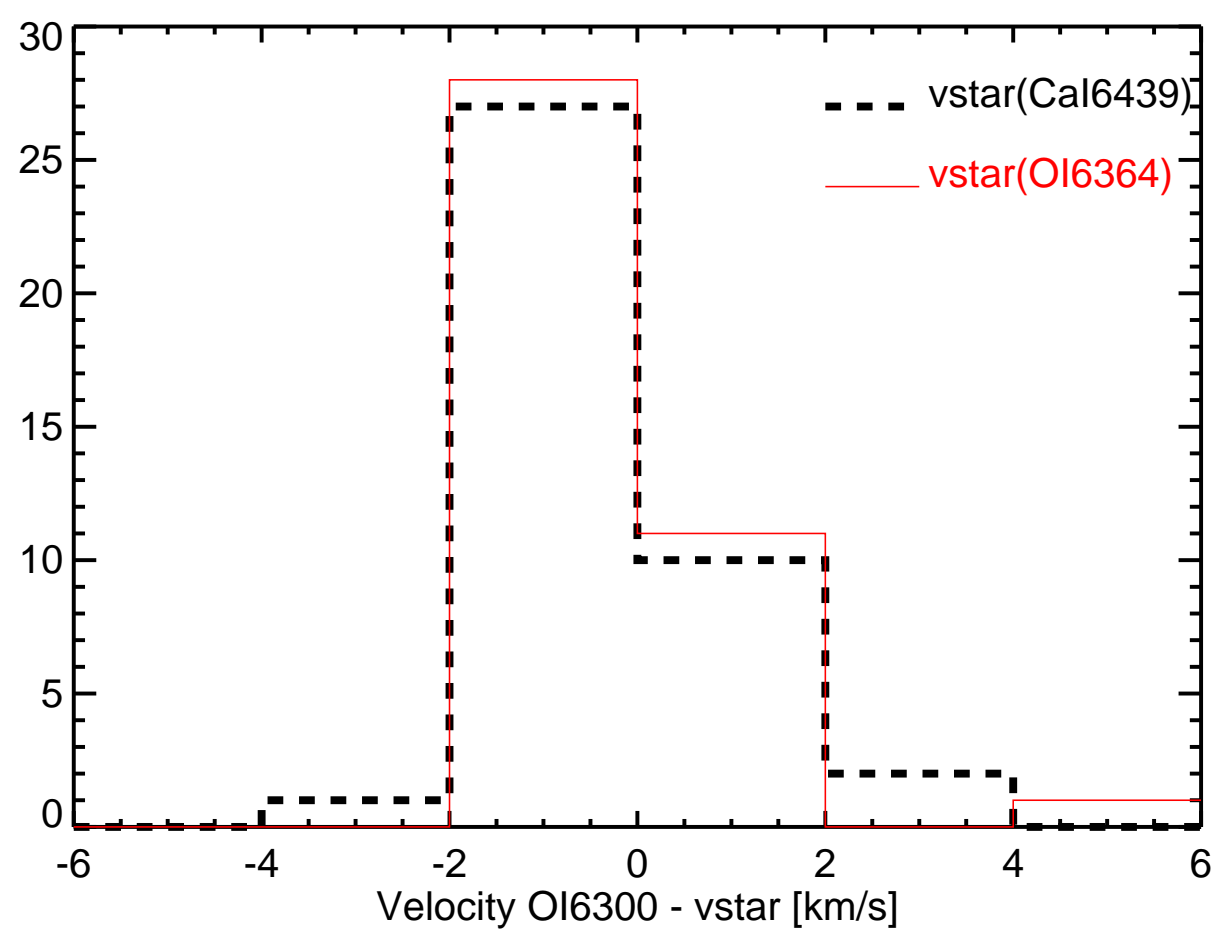

Fig. 5.- Histogram of the velocity shifts of the [O I] $6300 \AA$ line with respect to the stellar velocity measured via the photospheric CaI 6439 (black dashed line) and the [O I] $6364 \AA$ (red solid line) lines. The CaI $6439 \AA$ and the [O I] $6364 \AA$ fall on the same echelle order while the [O I] $6300 \AA$ line falls on a different echelle order. The fact that the two histograms look very similar and have the same median and standard deviation demonstrate that the [O I] $6300 \AA$ line is centered at the stellar velocity and the small blueshift between the [O I] 6300 and the CaI $6439 \AA$ line is just due to small differences in the dispersion solutions of the two echelle orders. 


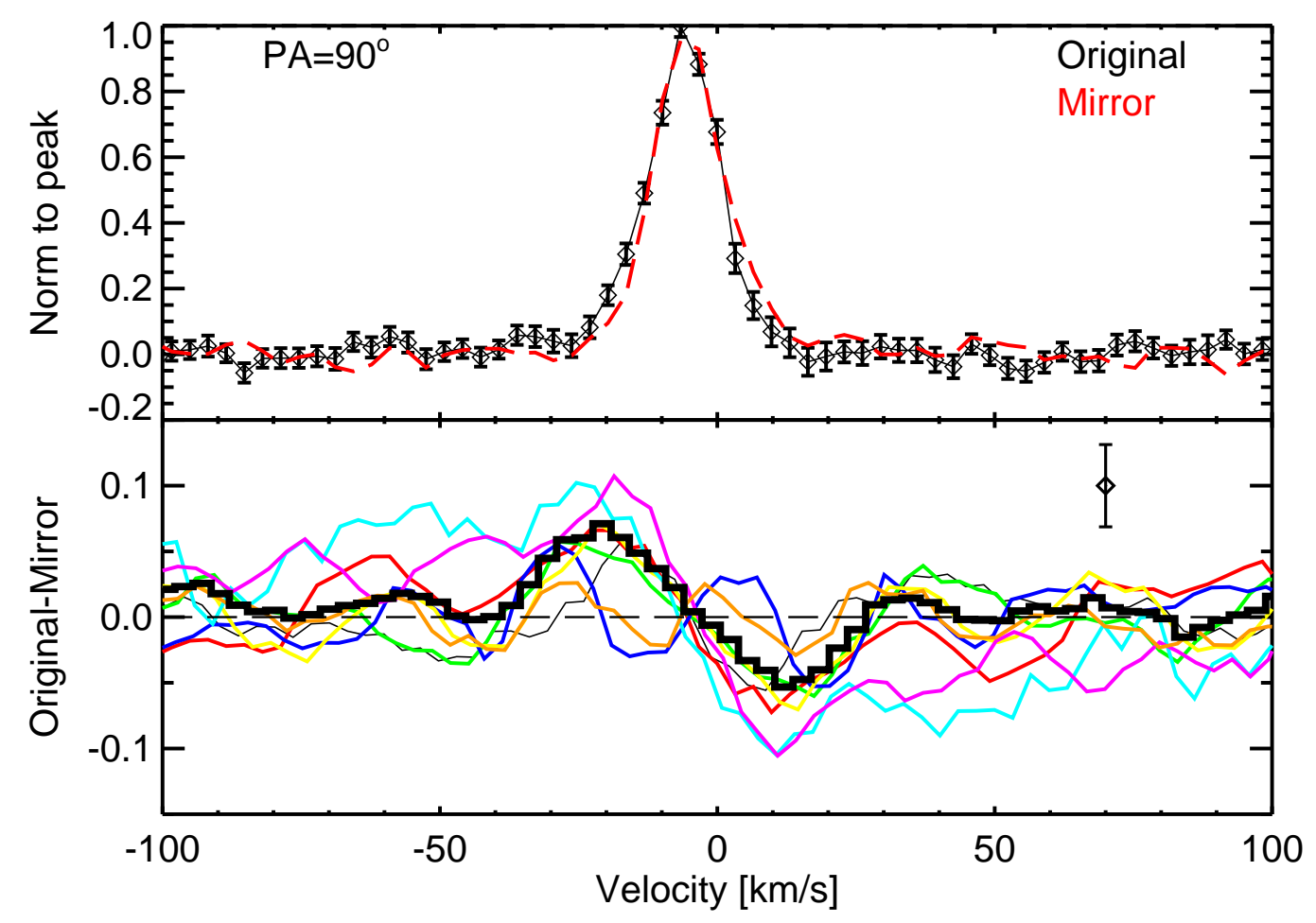

Fig. 6.- [Ne II] line profiles from TW Hya. The upper panel gives the original (black) and mirror-shifted (red) profile for $\mathrm{PA}=90^{\circ}$. Note that the continuum is subtracted and the emission is normalized to the peak. The lower panel shows the difference curves (originalmirror) for all eight PAs. The color coding is as follows: light black for PA $180^{\circ}$, red for $90^{\circ}$, green for $0^{\circ}$, blue for $270^{\circ}$, yellow for $153^{\circ}$, cyan for $63^{\circ}$, violet for $243^{\circ}$, and orange for $333^{\circ}$. The average difference curve is overplotted using a thick black line. Note that most difference curves (as well as the average difference curve) have an excess on the blue side, peaking at about $-20 \mathrm{~km} / \mathrm{s}$. This shows that the line is asymmetric with a blue wing due to photoevaporating gas accelerating toward the observer. 


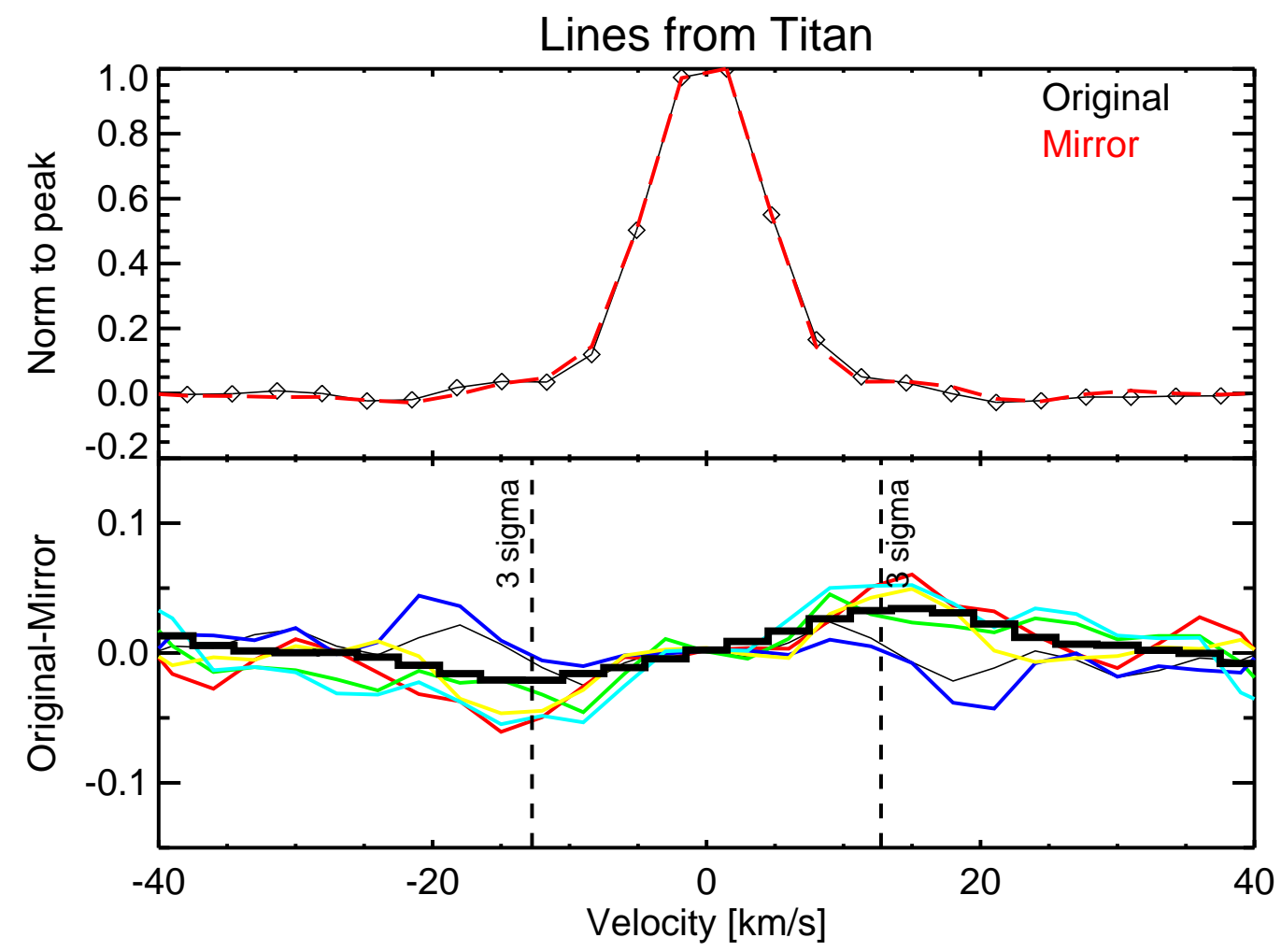

Fig. 7.- Line profiles from Titan (PID 083.C-0883). The continuum near the line is removed and lines are normalized to the peak emission as for TW Hya (also note the same y scale as in Fig. 6). Lower panel: difference curves (original-mirror profiles) for 3 emission lines from two different spectra of Titan. This figure shows that the asymmetry seen in the [Ne II] line profiles of TW Hya is not an instrumental artifact. 


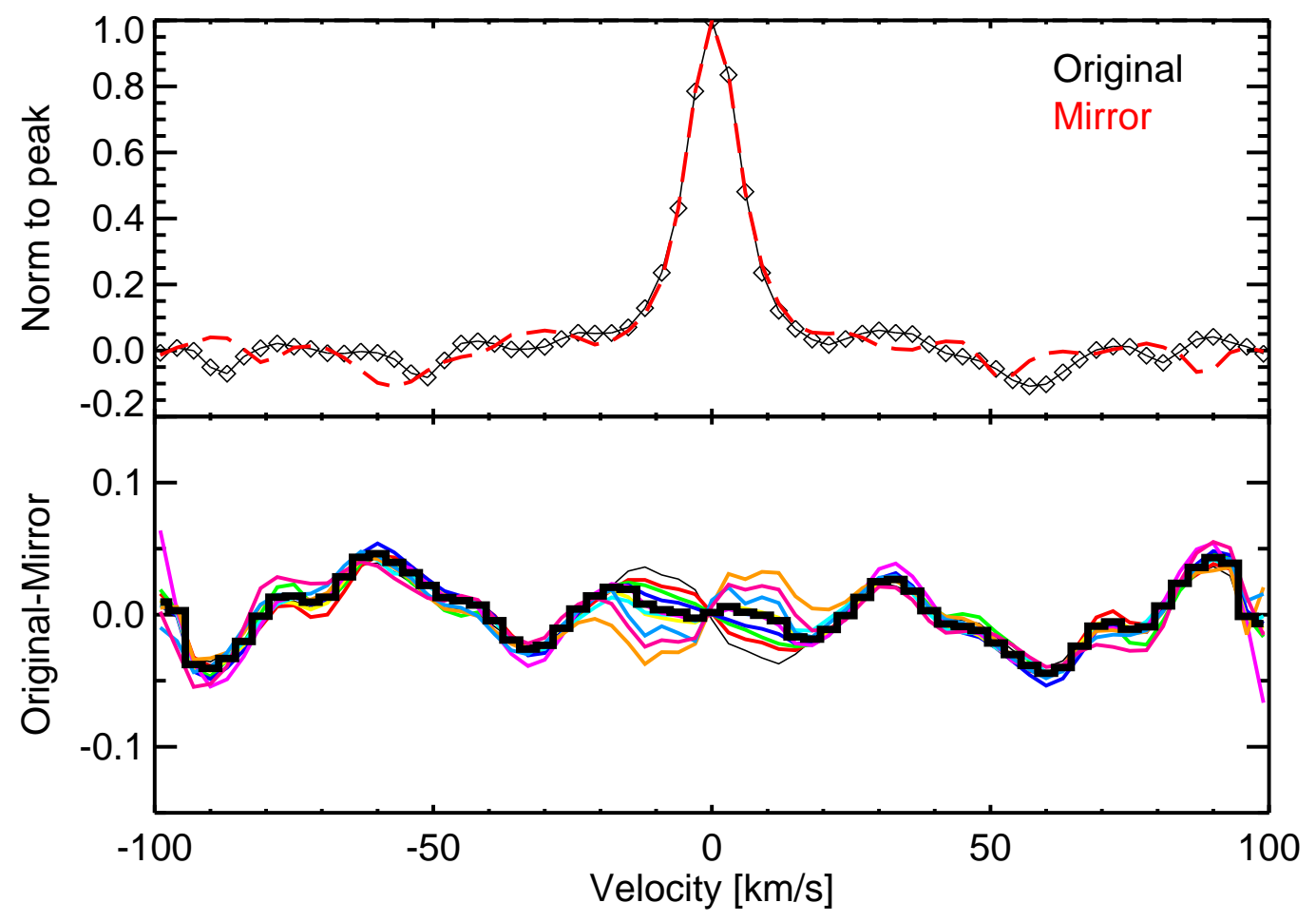

Fig. 8.- Asymmetry analysis for the [O I] $6300 \AA$ emission lines of TW Hya. The upper panel gives the original (black) and mirror-shifted (red) profile from one of the optical spectra obtained by Alencar \& Batalha (2002). The lower panel shows the difference curves (originalmirror) from the 10 spectra obtained in February 2000 (Alencar \& Batalha 2002), different colors are for spectra obtained in different days. Note that unlike the [Ne II] line at $12.81 \mu \mathrm{m}$ the $[\mathrm{O} \mathrm{I}]$ line at $6300 \AA$ is not blueshifted and has a symmetric profile. 


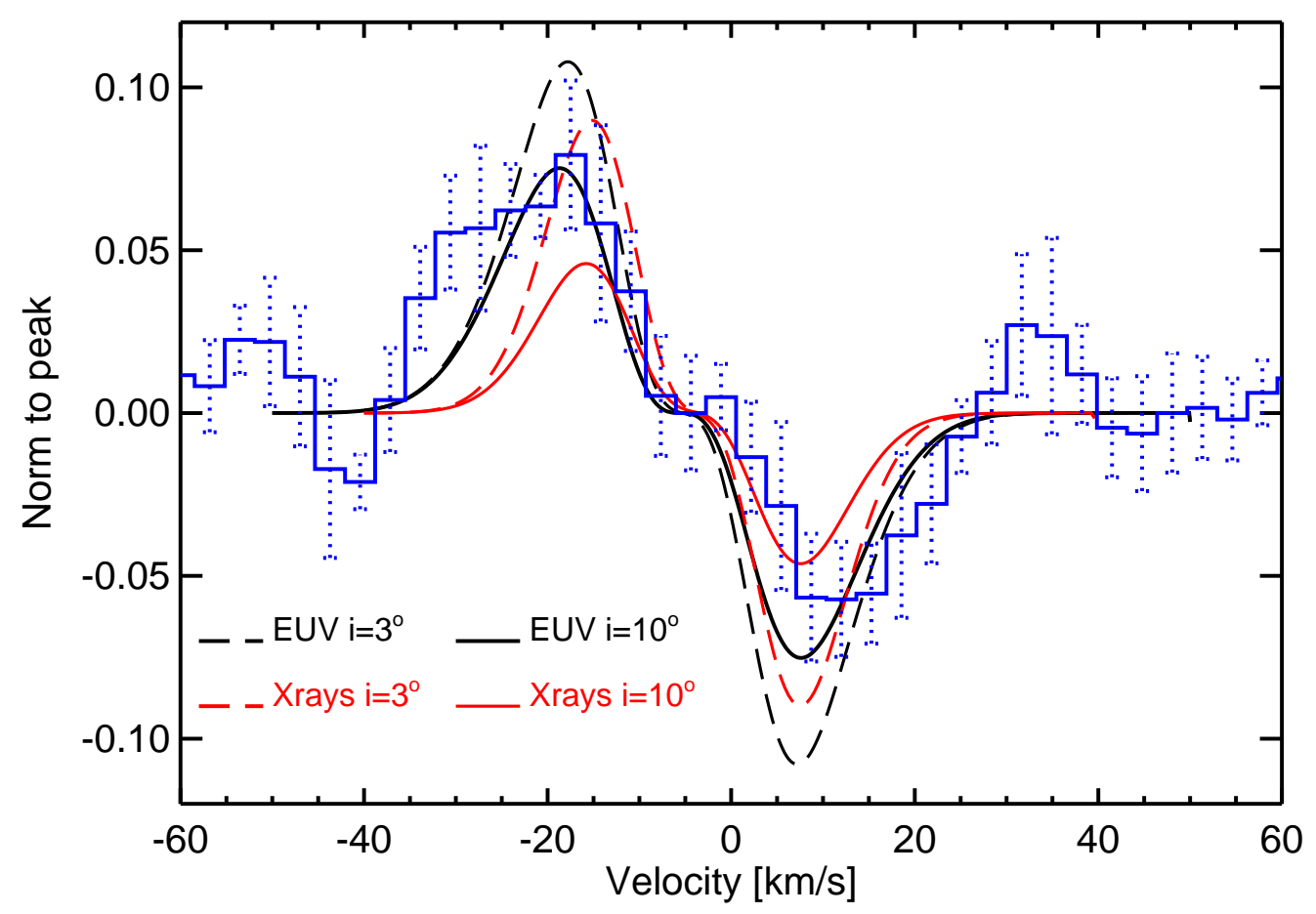

Fig. 9.- Comparison of difference curves (original-mirror profiles) for predicted and observed [Ne II] line profiles. Black solid and dashed lines are the difference curves from the EUV photoevaporation model, while red solid and dashed lines are from the Xray photoevaporation model for disks inclined by 10 and $3^{\circ}$ respectively. All models assume that the disk midplane is optically thick. The blue line is the median of the difference curves from the observed [Ne II] profiles of TW Hya (see also Fig. 6) with the standard deviation of the curves overplotted. 

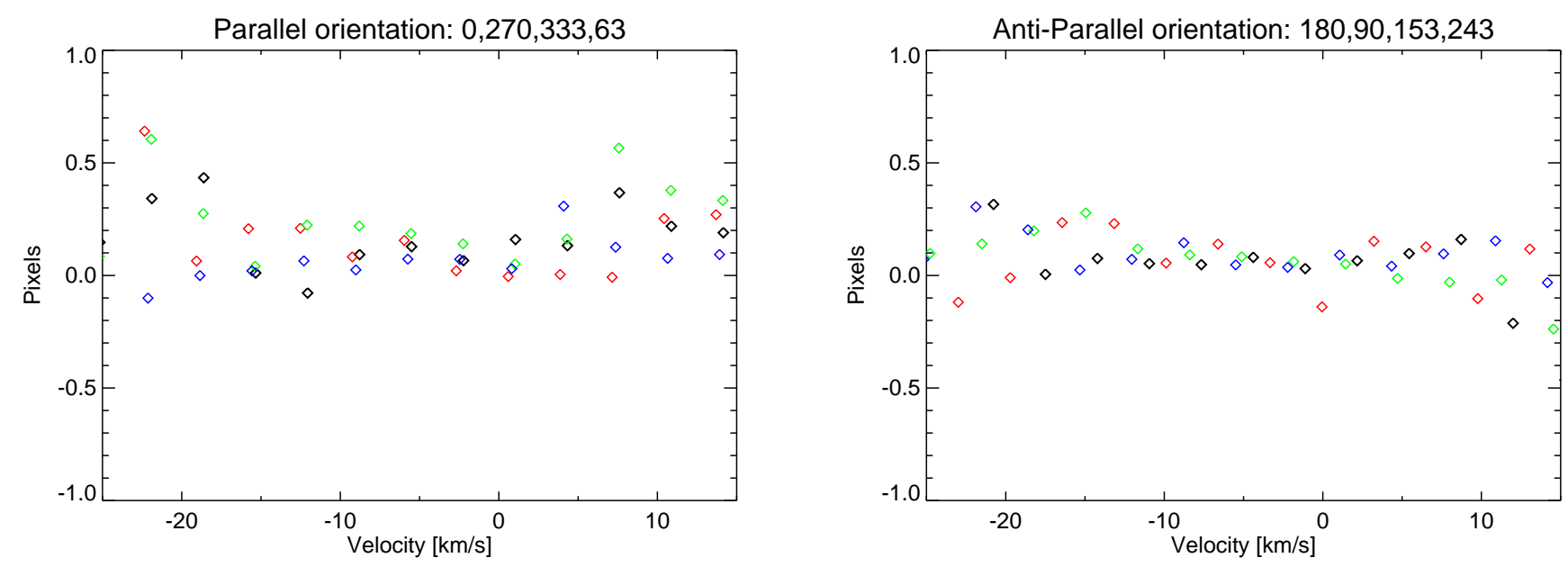

Fig. 10.- Centroid in pixels versus velocities in the stellocentric frame for the parallel (left panel) and anti-parallel (right panel) slit orientations. The color coding is as follows: black for 0 and $180^{\circ}$, red for 270 and $90^{\circ}$, green for 333 and $153^{\circ}$, and blue for 63 and $243^{\circ}$. 


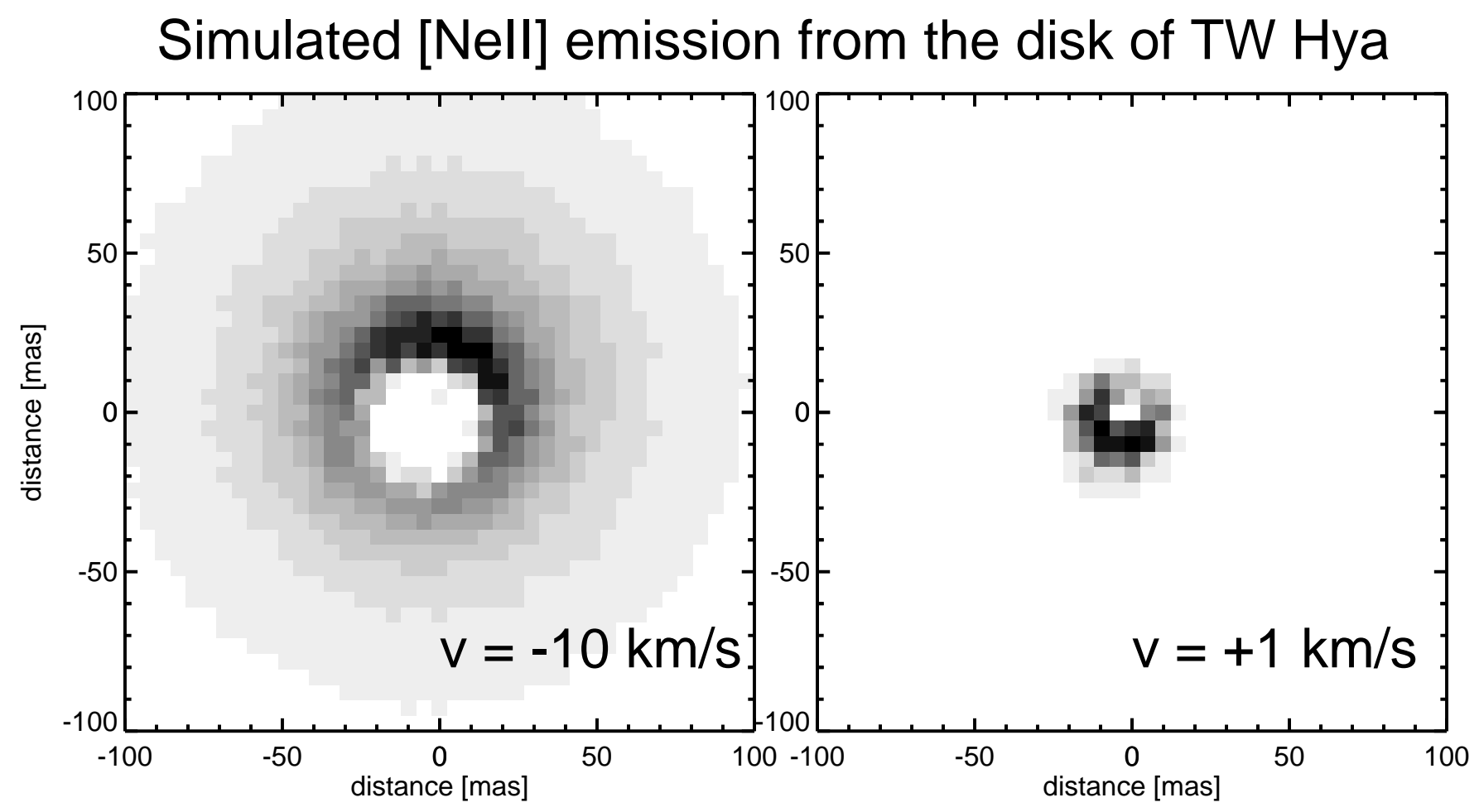

Fig. 11.- Modelled [Ne II] emission for two velocity channels, $-10 \mathrm{~km} / \mathrm{s}$ and $+1 \mathrm{~km} / \mathrm{s}$. The asymmetry is mainly due to the combination of disk inclination $\left(4^{\circ}\right)$ and Keplerian rotation. The wind is mainly symmetric but introduces deviations from the spectroastrometric Keplerian profiles, see text. The TW Hya disk position angle is $333^{\circ}$. On the left panel the black rim N-W is a factor of $\sim 3$ brighter than the grey emission S-E. On the right panel the contrast between the bright emission (now S-E) and the weaker emission (N-W) is about 5 but occurs at a much smaller spatial scale. These images are convolved with the VISIR PSF when simulating the spectro-astrometric signal. 


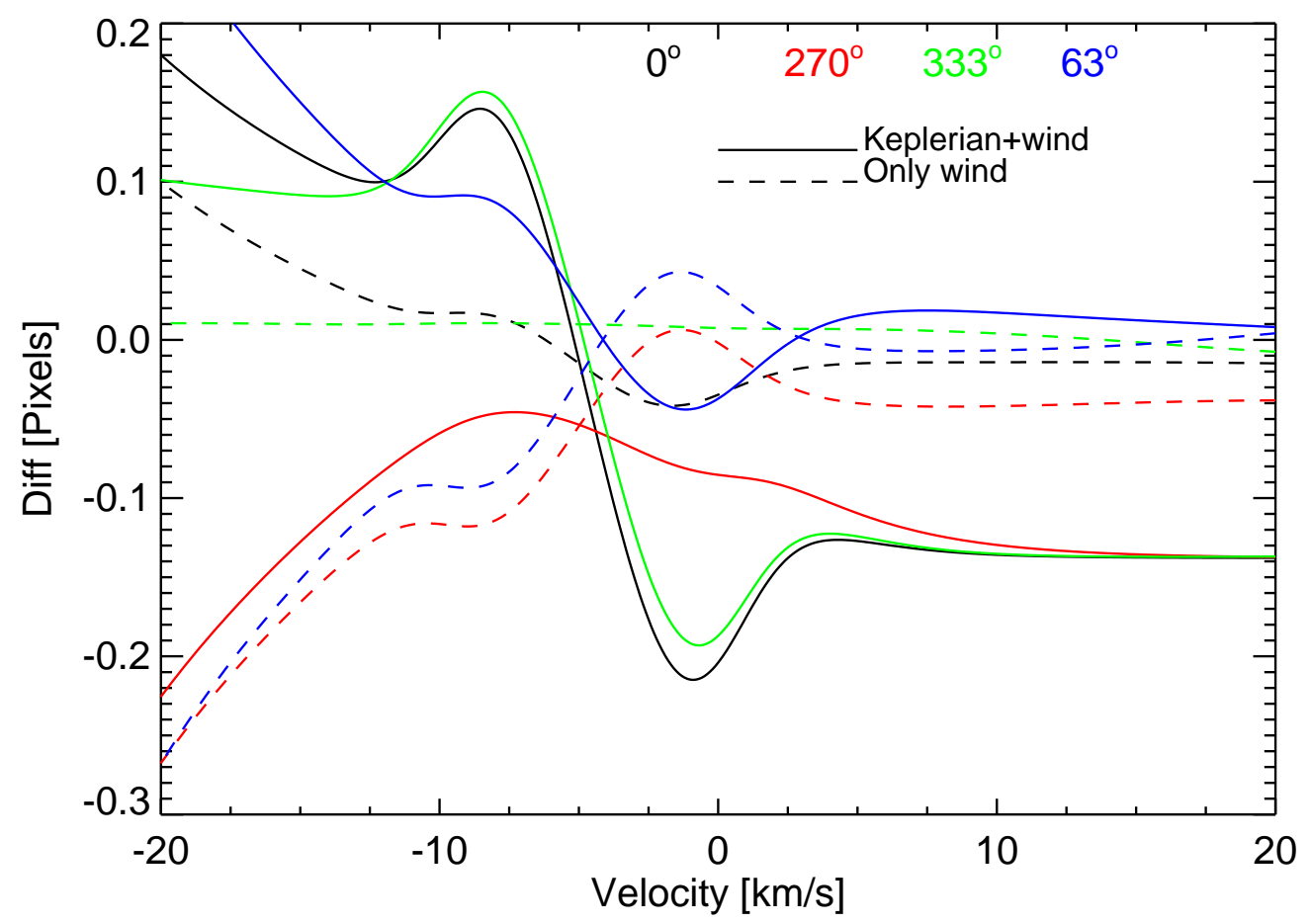

Fig. 12. - Spectroastrometric signal (parallel minus antiparallel centroids) predicted by a disk photoevaporated by stellar EUV photons (solid lines). Dashed lines show only the wind component, i.e. when Keplerian rotation is artificially set to zero. The plot shows that the largest spectroastrometric signal from the disk wind is expected for $\mathrm{PA}=63^{\circ}$, the slit orientation perpendicular to the disk major axis. One pixel on VISIR is 127 mas. 


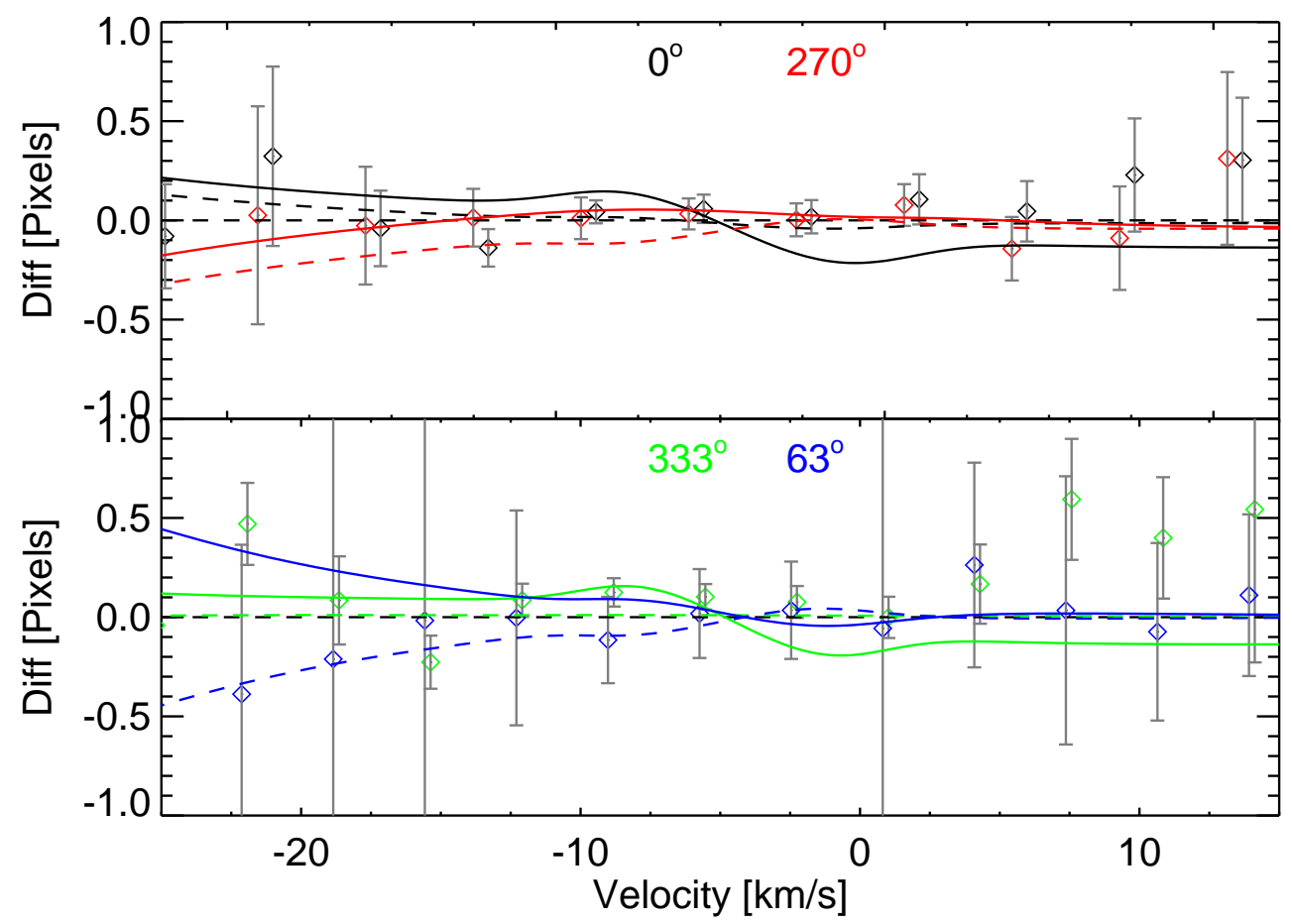

Fig. 13. - Spectroastrometric signal observed (diamonds) and predicted (lines, see Fig. 12 for color coding). The y-axis gives the difference in pixels between a position angle and its antiparallel position angle (e.g., $0^{\circ}$ corresponds to $0^{\circ}-180^{\circ}$ ). One pixel on VISIR is 127 mas, therefore the level of asymmetry we detect has a radial extension of $\sim 1.3 \mathrm{AU}$. 
Table 1. Summary of the TW Hya observations. The observing time (in U.T.), the airmass, and the heliocentric radial velocity corrections ( $\mathrm{v}_{\text {helio }}$ ) are given at the beginning and at the end of each spectroscopic exposure. On-source exposure times for TW Hya were set to $1 \mathrm{~h}$, for standard stars exposures varied between 240 and $360 \mathrm{~s}$. In the second night, we observed only one standard star for $\mathrm{PA}=333^{\circ}$.

\begin{tabular}{lcccccc}
\hline \hline $\begin{array}{l}\text { PA }^{\mathrm{a}} \\
\left(^{\circ}\right)\end{array}$ & $\begin{array}{c}\text { Date } \\
\text { yyyy-mm-dd }\end{array}$ & $\begin{array}{c}\text { U.T. } \\
\text { hh:mm }\end{array}$ & Airmass & $\begin{array}{c}\text { vhelio } \\
(\mathrm{km} / \mathrm{s})\end{array}$ & Calibrator & Airmass \\
\hline 0 & $2010-02-23$ & $02: 12 / 03: 41$ & $1.3 / 1.1$ & $11.53 / 11.40$ & HD 90957 & 1.3 \\
180 & $2010-02-23$ & $03: 54 / 05: 23$ & $1.1 / 1.0$ & $11.38 / 11.23$ & HD 90957 & 1.0 \\
270 & $2010-02-23$ & $06: 27 / 07: 54$ & $1.0 / 1.2$ & $11.11 / 10.97$ & HD 101666 & 1.0 \\
90 & $2010-02-23$ & $08: 25 / 09: 42$ & $1.3 / 1.5$ & $10.93 / 10.84$ & HD 101666 & 1.5 \\
\hline 333 & $2010-02-24$ & $02: 45 / 04: 15$ & $1.2 / 1.1$ & $11.11 / 10.97$ & HD 90957 & 1.2 \\
153 & $2010-02-24$ & $04: 28 / 05: 58$ & $1.0 / 1.0$ & $10.95 / 10.79$ & - & - \\
63 & $2010-02-24$ & $06: 24 / 07: 51$ & $1.0 / 1.2$ & $10.74 / 10.70$ & - & - \\
243 & $2010-02-24$ & $08: 07 / 09: 38$ & $1.2 / 1.7$ & $10.58 / 10.47$ & - & - \\
\hline
\end{tabular}

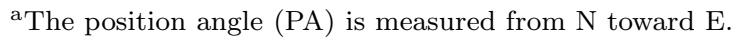

Table 2. Main properties of the TW Hya star/disk system inferred from other studies and utilized in this paper.

\begin{tabular}{|c|c|c|}
\hline Property & Value & Reference \\
\hline Stellar mass & $0.7 \mathrm{M}_{\odot}$ & 1 \\
\hline Distance & $51 \pm 4 \mathrm{pc}$ & 2 \\
\hline Disk inclination & $4.3 \pm 1^{\circ}, 7 \pm 1^{\circ}, 5-6^{\circ}$ & $3,4,5$ \\
\hline Disk PA & $332 \pm 10^{\circ}, 335 \pm 2^{\circ}$ & 3,5 \\
\hline Stellar ionizing flux & $10^{41} \mathrm{phot} / \mathrm{s}$ & 6 \\
\hline \multicolumn{3}{|c|}{ References. - (1) Muzerolle et al. 2000; (2) Mamajek } \\
\hline 2005; (3) Pontoppida & et al.|2008; (4) Qi et al. & $2004 ;(5)$ \\
\hline Hughes et al. 2011; ( & Pascucci \& Sterzik 2009 & \\
\hline
\end{tabular}


Table 3. Main parameters of the [Ne II] line and adjacent continuum for different position angles (PA).

\begin{tabular}{|c|c|c|c|c|c|}
\hline $\begin{array}{l}\mathrm{PA} \\
\left({ }^{\circ}\right)\end{array}$ & $\begin{array}{l}\text { EW } \\
(\AA)\end{array}$ & $\begin{array}{l}\text { FWHM } \\
(\mathrm{km} / \mathrm{s})\end{array}$ & $\begin{array}{c}\mathrm{v}_{\text {peak }} \\
(\mathrm{km} / \mathrm{s})\end{array}$ & $\begin{array}{c}\text { Line Flux } \\
\left(\times 10^{-14} \mathrm{erg} \mathrm{cm}^{-2} \mathrm{~s}^{-1}\right)\end{array}$ & $\begin{array}{l}\text { Continuum } \\
(\mathrm{Jy})\end{array}$ \\
\hline 0 & $43 \pm 1$ & $15.2 \pm 0.4$ & $-5.0 \pm 0.2$ & $2.6 \pm 0.1$ & 0.3 \\
\hline 180 & $47.1 \pm 0.8$ & $16.9 \pm 0.4$ & $-4.9 \pm 0.1$ & $6.5 \pm 0.2$ & 0.8 \\
\hline 270 & $44 \pm 1$ & $15.0 \pm 0.4$ & $-4.8 \pm 0.2$ & $4.4 \pm 0.1$ & 0.6 \\
\hline 90 & $36 \pm 1$ & $15.8 \pm 0.5$ & $-5.9 \pm 0.2$ & $3.0 \pm 0.1$ & 0.5 \\
\hline 333 & $47 \pm 1$ & $15.9 \pm 0.4$ & $-6.2 \pm 0.2$ & $3.2 \pm 0.1$ & 0.4 \\
\hline 153 & $47.7 \pm 0.8$ & $16.6 \pm 0.3$ & $-3.9 \pm 0.3$ & - & - \\
\hline 63 & $30 \pm 1$ & $17.5 \pm 0.7$ & $-7.5 \pm 0.3$ & - & - \\
\hline 243 & $35 \pm 1$ & $17.2 \pm 0.6$ & $-3.9 \pm 0.3$ & - & - \\
\hline
\end{tabular}

Note. - Errors on the EWs are computed using a Monte Carlo approach: we added a normally distributed noise to the spectrum (following the error at each wavelength) and give as error the standard deviation of the EW distribution of 1000 such spectra. The errors on the FWHM, peak emission, and line flux are computed from the gaussian fit to the [Ne II] line taking into account the uncertainty at each wavelength. Note that the absolute radial velocity accuracy reachable with VISIR is $\sim 2 \mathrm{~km} / \mathrm{s}$ (Sect. 3.1), larger than the uncertainties on the [Ne II] peak velocities derived from the gaussian fit. 
Table 4. Line EWs and upper limits for other optical lines predicted to trace the photoevaporative wind.

\begin{tabular}{lcccccc}
\hline \hline Species & $\begin{array}{c}\text { Wavelength } \\
(\AA)\end{array}$ & $\begin{array}{c}\mathrm{A}_{\mathrm{ul}}{ }^{\mathrm{a}} \\
\left(\mathrm{s}^{-1}\right)\end{array}$ & Terms & $\begin{array}{c}\text { EW } \\
(\AA)\end{array}$ & veiling & $\begin{array}{c}\text { Luminosity } \\
(\mathrm{A})\end{array}$ \\
\hline OI & 5577.3387 & $1.26(0)$ & ${ }^{1} \mathrm{D}-{ }^{1} \mathrm{~S}$ & 0.09 & 0.5 & $1.5(-6)$ \\
OI & 6300.304 & $5.65(-3)$ & ${ }^{3} \mathrm{P}-{ }^{1} \mathrm{D}$ & 0.47 & 0.6 & $1.1(-5)$ \\
OI & 6363.777 & $1.82(-3)$ & ${ }^{3} \mathrm{P}-{ }^{1} \mathrm{D}$ & 0.15 & 0.4 & $3.2(-6)$ \\
OII & 7330.73 & $5.34(-2)$ & ${ }^{2} \mathrm{D}-{ }^{2} \mathrm{P}$ & $<0.04$ & 0.5 & $<9.1(-7)$ \\
MgI & 4571.0956 & $2.54(2)$ & ${ }^{1} \mathrm{~S}-{ }^{3} \mathrm{P}$ & 0.08 & 0.5 & $6.4(-7)$ \\
NII & 6583.460 & $2.92(-3)$ & ${ }^{3} \mathrm{P}-{ }^{1} \mathrm{D}$ & $<0.007$ & 0.4 & $<1.5(-7)$ \\
SII & 4068.600 & $3.41(-1)$ & ${ }^{4} \mathrm{~S}-{ }^{2} \mathrm{P}$ & 0.09 & 2 & $1.4(-6)$ \\
SII & 4076.349 & $1.34(-1)$ & ${ }^{4} \mathrm{~S}-{ }^{2} \mathrm{P}$ & $<0.03$ & 2 & $<4.8(-7)$ \\
SII & 6716.440 & $4.66(-4)$ & ${ }^{4} \mathrm{~S}-{ }^{2} \mathrm{D}$ & $<0.01$ & 0.5 & $<2.3(-7)$ \\
SII & 6730.810 & $4.26(-4)$ & ${ }^{4} \mathrm{~S}-{ }^{2} \mathrm{D}$ & $<0.008$ & 0.6 & $<1.9(-7)$ \\
\hline
\end{tabular}

${ }^{a}$ The transition probability from NIST and the line luminosity are given as a(b) meaning $a \times 10^{b}$.

Note. - Wavelengths are in air. EWs, veilings, and corresponding line luminosities are median values. Uncertainties on the measured EWs are about $\sim 10 \%$. Because of night-to-night variations in the source brightness and in the veiling the luminosities reported here have an uncertainty of $\sim 50 \%$ (see Sect. 4.2). 


\section{REFERENCES}

Adams, F. C., Hollenbach, D., Laughlin, G., \& Gorti, U. 2004, ApJ, 611, 360

Akeson, R. L., et al. 2011, ApJ, 728, 96

Alencar, S. H. P., \& Batalha, C. 2002, ApJ, 571, 378

Alexander, R. D. 2008, MNRAS, 391, L64

Alexander, R. D., Clarke, C. J., \& Pringle, J. E. 2006a, MNRAS, 369, 216

-. 2006b, MNRAS, 369, 229

Armitage, P. J. 2010, ArXiv e-prints

Bailey, J. 1998, MNRAS, 301, 161

Calvet, N., D’Alessio, P., Hartmann, L., Wilner, D., Walsh, A., \& Sitko, M. 2002, ApJ, 568, 1008

Calvet, N., Hartmann, L., \& Strom, S. E. 2000, Protostars and Planets IV, 377

Cieza, L. A., Swift, J. J., Mathews, G. S., \& Williams, J. P. 2008, ApJ, 686, L115

Clarke, C. J., Gendrin, A., \& Sotomayor, M. 2001, MNRAS, 328, 485

Currie, T., Lada, C. J., Plavchan, P., Robitaille, T. P., Irwin, J., \& Kenyon, S. J. 2009, ApJ, 698, 1

Dullemond, C. P., Hollenbach, D., Kamp, I., \& D’Alessio, P. 2007, Protostars and Planets V, 555

Ercolano, B., \& Clarke, C. J. 2010, MNRAS, 402, 2735

Ercolano, B., Drake, J. J., Raymond, J. C., \& Clarke, C. C. 2008, ApJ, 688, 398 
Ercolano, B., \& Owen, J. E. 2010, MNRAS, 406, 1553

Font, A. S., McCarthy, I. G., Johnstone, D., \& Ballantyne, D. R. 2004, ApJ, 607, 890

Glassgold, A. E., Najita, J. R., \& Igea, J. 2007, ApJ, 656, 515

Gorti, U., Dullemond, C. P., \& Hollenbach, D. 2009, ApJ, 705, 1237

Gorti, U., \& Hollenbach, D. 2009, ApJ, 690, 1539

Gray, D. F. 1980, ApJ, 235, 508

Hartigan, P., Edwards, S., \& Ghandour, L. 1995, ApJ, 452, 736

Hartmann, L., Calvet, N., Gullbring, E., \& D’Alessio, P. 1998, ApJ, 495, 385

Hayashi, C., Nakazawa, K., \& Nakagawa, Y. 1985, in Protostars and Planets II, ed. D. C. Black \& M. S. Matthews, 1100-1153

Herczeg, G. J., Najita, J. R., Hillenbrand, L. A., \& Pascucci, I. 2007, ApJ, 670, 509

Hollenbach, D., \& Gorti, U. 2009, ApJ, 703, 1203

Hollenbach, D., Johnstone, D., Lizano, S., \& Shu, F. 1994, ApJ, 428, 654

Hollenbach, D., \& McKee, C. F. 1989, ApJ, 342, 306

Hollenbach, D. J., Yorke, H. W., \& Johnstone, D. 2000, Protostars and Planets IV, 401

Horne, K. 1986, PASP, 98, 609

Hughes, A. M., Wilner, D. J., Andrews, S. M., Qi, C., \& Hogerheijde, M. R. 2011, ApJ, 727,85

Käufl, H. U. 2006, in Planetary Nebulae Beyond the Milky Way, ed. L. Stanghellini, J. R. Walsh, \& N. G. Douglas, 201-+ 
Kim, K. H., et al. 2009, ApJ, 700, 1017

Lagage, P. O., et al. 2004, The Messenger, 117, 12

Liffman, K. 2003, PASA, 20, 337

Lubow, S. H., \& D’Angelo, G. 2006, ApJ, 641, 526

Mamajek, E. E. 2005, ApJ, 634, 1385

Muzerolle, J., Calvet, N., Briceño, C., Hartmann, L., \& Hillenbrand, L. 2000, ApJ, 535, L47

Muzerolle, J., Hartmann, L., \& Calvet, N. 1998, AJ, 116, 455

Najita, J. R., Carr, J. S., Strom, S. E., Watson, D. M., Pascucci, I., Hollenbach, D., Gorti, U., \& Keller, L. 2010, ApJ, 712, 274

Natta, A., Testi, L., Calvet, N., Henning, T., Waters, R., \& Wilner, D. 2007, Protostars and Planets V, 767

Owen, J. E., Ercolano, B., \& Clarke, C. J. 2011, MNRAS, 412, 13

Owen, J. E., Ercolano, B., Clarke, C. J., \& Alexander, R. D. 2010, MNRAS, 401, 1415

Pascucci, I., \& Sterzik, M. 2009, ApJ, 702, 724

Pascucci, I., \& Tachibana, S. 2010, The Clearing of Protoplanetary Disks and of the Protosolar Nebula (Apai, D. A. \& Lauretta, D. S.), 263-298

Pontoppidan, K. M., Blake, G. A., van Dishoeck, E. F., Smette, A., Ireland, M. J., \& Brown, J. 2008, ApJ, 684, 1323

Qi, C., et al. 2004, ApJ, 616, L11 
Ratzka, T., Leinert, C., Henning, T., Bouwman, J., Dullemond, C. P., \& Jaffe, W. 2007, A\&A, 471, 173

Rothman, L. S., \& Gordon, I. E. 2009, in 64th International Symposium On Molecular Spectroscopy

Rucinski, S. M., et al. 2008, MNRAS, 391, 1913

Sicilia-Aguilar, A., Henning, T., \& Hartmann, L. W. 2010, ApJ, 710, 597

Weintraub, D. A., Kastner, J. H., \& Bary, J. S. 2000, ApJ, 541, 767

Whelan, E., \& Garcia, P. 2008, in Lecture Notes in Physics, Berlin Springer Verlag, Vol. 742, Jets from Young Stars II, ed. F. Bacciotti, L. Testi, \& E. Whelan, 123-+

Yasui, C., Kobayashi, N., Tokunaga, A. T., Saito, M., \& Tokoku, C. 2009, ApJ, 705, 54

Zhu, Z., Nelson, R. P., Hartmann, L., Espaillat, C., \& Calvet, N. 2011, ApJ, 729, 47 